\title{
CLUMPY GALAXIES IN CANDELS. I. THE DEFINITION OF UV CLUMPS AND THE FRACTION OF CLUMPY GALAXIES AT $0.5<z<3$
}

\author{
Yicheng Guo $^{1}$, Henry C. Ferguson ${ }^{2}$, Eric F. Bell ${ }^{3}$, David C. Koo ${ }^{1}$, Christopher J. Conselice ${ }^{4}$, Mauro Giavalisco ${ }^{5}$, \\ Susan Kassin ${ }^{2}$, Yu Lu $^{6}$, Ray Lucas ${ }^{2}$, Nir Mandelker ${ }^{7}$, Daniel M. McIntosh ${ }^{8}$, Joel R. Primack ${ }^{9}$, Swara Ravindranath $^{2}$, \\ Guillermo Barro ${ }^{1}$, Daniel Ceverino ${ }^{10}$, Avishai Dekel ${ }^{7}$, Sandra M. Faber ${ }^{1}$, Jerome J. Fang ${ }^{1}$, Anton M. Koekemoer ${ }^{2}$, \\ Kai NoesKe ${ }^{2}$, Marc Rafelski ${ }^{11,12}$, and Amber Straughn ${ }^{11}$ \\ ${ }^{1}$ UCO/Lick Observatory, Department of Astronomy and Astrophysics, University of California, Santa Cruz, CA, USA; ycguo@ucolick.org \\ ${ }^{2}$ Space Telescope Science Institute, Baltimore, MD, USA \\ ${ }^{3}$ Department of Astronomy, University of Michigan, Ann Arbor, MI, USA \\ ${ }^{4}$ School of Physics and Astronomy, University of Nottingham, University Park, Nottingham NG7 2RD, UK \\ ${ }_{5}$ Department of Astronomy, University of Massachusetts, Amherst, MA, USA \\ ${ }^{6}$ Kavli Institute for Particle Astrophysics and Cosmology, Stanford, CA, USA \\ ${ }^{7}$ Center for Astrophysics and Planetary Science, Racah Institute of Physics, The Hebrew University, Jerusalem 91904 Israel \\ ${ }^{8}$ Department of Physics and Astronomy, University of Missouri-Kansas City, Kansas City, MO, USA \\ ${ }^{9}$ Department of Physics, University of California, Santa Cruz, CA, USA \\ ${ }^{10}$ Departamento de Física Teórica, Universidad Autónoma de Madrid, E-28049 Madrid, Spain \\ ${ }^{11}$ Astrophysics Science Division, Goddard Space Flight Center, Code 665, Greenbelt, MD 20771, USA \\ Received 2014 July 14; accepted 2014 November 21; published 2015 February 6
}

\begin{abstract}
Although giant clumps of stars are thought to be crucial to galaxy formation and evolution, the most basic demographics of clumps are still uncertain, mainly because the definition of clumps has not been thoroughly discussed. In this paper, we carry out a study of the basic demographics of clumps in star-forming galaxies at $0.5<z<3$, using our proposed physical definition that UV-bright clumps are discrete star-forming regions that individually contribute more than $8 \%$ of the rest-frame UV light of their galaxies. Clumps defined this way are significantly brighter than the $\mathrm{H}$ II regions of nearby large spiral galaxies, either individually or blended, when physical spatial resolution and cosmological dimming are considered. Under this definition, we measure the fraction of star-forming galaxies that have at least one off-center clump $\left(f_{\text {clumpy }}\right)$ and the contributions of clumps to the rest-frame UV light and star formation rate (SFR) of star-forming galaxies in the CANDELS/GOODS-S and UDS fields, where our mass-complete sample consists of 3239 galaxies with axial ratio $q>0.5$. The redshift evolution of $f_{\text {clumpy }}$ changes with the stellar mass $\left(M_{*}\right)$ of the galaxies. Low-mass $\left(\log \left(M_{*} / M_{\odot}\right)<9.8\right)$ galaxies keep an almost constant $f_{\text {clumpy }}$ of $\sim 60 \%$ from $z \sim 3$ to $z \sim 0.5$. Intermediate-mass and massive galaxies drop their $f_{\text {clumpy }}$ from $55 \%$ at $z \sim 3$ to $40 \%$ and $15 \%$, respectively, at $z \sim 0.5$. We find that (1) the trend of disk stabilization predicted by violent disk instability matches the $f_{\text {clumpy }}$ trend of massive galaxies; (2) minor mergers are a viable explanation of the $f_{\text {clumpy }}$ trend of intermediate-mass galaxies at $z<1.5$, given a realistic observability timescale; and (3) major mergers are unlikely responsible for the $f_{\text {clumpy }}$ trend in all masses at $z<1.5$. The clump contribution to the rest-frame UV light of star-forming galaxies shows a broad peak around galaxies with $\log \left(M_{*} / M_{\odot}\right) \sim 10.5$ at all redshifts. The clump contribution in the intermediate-mass and massive galaxies is possibly linked to the molecular gas fraction of the galaxies. The clump contribution to the SFR of star-forming galaxies, generally around 4\%-10\%, also shows dependence on the galaxy $M_{*}$, but for a given galaxy $M_{*}$, its dependence on the redshift is mild.
\end{abstract}

Key words: galaxies: evolution - galaxies: formation - galaxies: irregular - galaxies: starburst galaxies: star formation - galaxies: structure

\section{INTRODUCTION}

The emergence of facilities with high sensitivity and high resolution, e.g., HST/ACS, NICMOS, and WFC3, enables astronomers to resolve galaxy morphology and structure to $\mathrm{kpc}$ scale to study the properties of galactic sub-structures at high redshift (e.g., Elmegreen \& Elmegreen 2005; Elmegreen et al. 2007, 2009a, 2009b; Gargiulo et al. 2011; Szomoru et al. 2011; Guo et al. 2011, 2012). An important observational feature of high-redshift star-forming galaxies (SFGs) is the existence of giant kpc-scale clumps of stars or star formation activities (e.g., Conselice et al. 2004; Elmegreen \& Elmegreen 2005; Elmegreen et al. 2007, 2009a; Bournaud et al. 2008; Genzel et al. 2008, 2011; Förster Schreiber et al. 2011; Guo et al. 2012; Wuyts et al. 2012), which are unusual in massive low-redshift galaxies.

\footnotetext{
${ }^{12}$ NASA Postdoctoral Program Fellow.
}

The giant clumps are mostly identified in the deep and highresolution rest-frame UV images (e.g., Elmegreen \& Elmegreen 2005; Elmegreen et al. 2007; Guo et al. 2012) and rest-frame optical images (e.g., Elmegreen et al. 2009a; Förster Schreiber et al. 2011). They are also seen in the rest-frame optical line emission from NIR integral field spectroscopy (e.g., Genzel et al. 2008, 2011) or CO line emission of lensed galaxies (e.g., Jones et al. 2010; Swinbank et al. 2010). The typical stellar mass $\left(M_{*}\right)$ of clumps is $10^{7}-10^{9} M_{\odot}$ (e.g., Elmegreen et al. 2007; Guo et al. 2012), and the typical size is $\sim 1 \mathrm{kpc}$ or less (e.g., Elmegreen et al. 2007; Förster Schreiber et al. 2011; Livermore et al. 2012). The clumps have blue UV-optical colors and are shown to be regions with enhanced specific star formation rates (SSFR), which are higher than that of their surrounding areas by a factor of several (e.g., Guo et al. 2012; Wuyts et al. 2012, 2013). Both morphological analysis (e.g., the Sérsic models; Elmegreen et al. 2007) and gas kinematic analysis (e.g., $\mathrm{H} \alpha$ 
velocity maps; Genzel et al. 2008, 2011) show that many clumpy galaxies have underlying disks.

Although clumps are thought to be important laboratories to test our knowledge of star formation, feedback, and galactic structure formation, the definition of "clump" has not been thoroughly discussed. Clumps were originally defined through the appearance of galaxies by visual inspection (e.g., Cowie et al. 1995; van den Bergh et al. 1996; Elmegreen et al. 2004, 2007; Elmegreen \& Elmegreen 2005). Visual definitions are, however, subjective and hard to reproduce. More and more studies have begun to automate the clump detection (e.g., Conselice 2003; Conselice et al. 2004; Förster Schreiber et al. 2011; Guo et al. 2012; Wuyts et al. 2012; Murata et al. 2014). Although these automated detections are easier to reproduce and to apply to large samples, most of them define clumps based on the appearance of galaxies, namely, the intensity contrast between the peak and the local background in galaxy images. The biggest problem of such definitions is that the appearance of even the same type of galaxies changes with the sensitivity and resolution of observations. Therefore, each of such definitions of clumps is actually bound to a given observation, which makes comparisons between different observations difficult. As a result, there are still large uncertainties in the most basic demographics of clumps: what fraction of SFGs have clumps, and what fraction of the total star formation occurs in clumps.

The measurement of the fraction of clumpy galaxies in the overall sample of SFGs ( $\left.f_{\text {clumpy }}\right)$ shows a large dispersion in literature. Ravindranath et al. (2006) claimed that clumpy galaxies are about $30 \%$ of the population at $z \sim 3$, while Elmegreen et al. (2007) argued that the dominant morphology for $z \gtrsim 2$ starbursts is clumpy galaxies. Guo et al. (2012) found a high $f_{\text {clumpy }} \sim$ $67 \%$ for SFGs at $z \sim 2$ in HUDF. However, their sample contains only 15 galaxies, which may be biased toward bright, blue, and large galaxies because they include only spectroscopically observed galaxies. Wuyts et al. (2012) measured the fraction of clumpy galaxies in a mass-complete sample of SFGs at $z \sim 2$ by using multi-waveband images and $M_{*}$ maps. They found that the clumpy fraction depends sensitively on the light/mass map used to identify the regions with excess surface brightness and decreases from about $75 \%$ for galaxies selected through restframe $2800 \AA$ images to about $40 \%$ for those selected through rest-frame $V$-band images or $M_{*}$ maps.

The clump contribution to the UV light and star formation rate (SFR) of the galaxies is closely related to the physics that drives galaxy formation and evolution, e.g., gas accretion rate, gas fraction, and star formation efficiency. For example, if clumps are formed in-situ through the violent disk instability (VDI; Dekel et al. 2009b) in gas-rich rotating disks that are perturbed by the accreted gas inflow, the clump contribution is expected to drop from high redshift to low redshift, because the cosmic cold gas accretion quickly declines with the cosmic time (e.g., Kereš et al. 2005; Dekel et al. 2009a). Wuyts et al. (2012, 2013), using color/SFR excess to identify clumpy regions, found the clump contribution to the cosmic SFR decreasing from $z=2.5$ to $z=1$, consistent with the above prediction. On the other hand, Mandelker et al. (2014), using gas maps to identify clumps, found that the clump contribution to SFR in the Ceverino et al. (2010) numerical simulations is almost flat, if not increasing, from $z=3$ to $z=1$. A possible reason for the discrepancy is that the above two studies did not define clumps in the same way. In fact, Moody et al. (2014) found that the one-to-one correspondence among the clumps defined through gas, young stars, and mass is poor. To unify the current rapid emergence of multi-wavelength observations of clumps as well as the stateof-the-art numerical simulations, it is crucial to have a more physical definition of "clump" to move the studies of clumps and clumpy galaxies forward.

In this paper, we propose a definition of clumps based on their intrinsic rest-frame UV properties and present a comprehensive measurement of $f_{\text {clumpy }}$ and its variation with redshift and $M_{*}$, exploiting the advantage of high resolution and deep sensitivity of HST/ACS and WFC3 images in the CANDELS/GOODS-S and UDS fields. We also measure the clump contribution to the rest-frame UV light and SFR of SFGs.

The paper is organized as follows. The data and sample selection are presented in Section 2. In Section 3, we start our clump definition by the traditional way of detecting discrete star-forming regions through the intensity contrast between the peak and background of galaxy images. We use an automated algorithm to detect the star-forming regions in the same restframe UV bands across a wide redshift range of $0.5<z<$ 3.0. In Section 4, we measure the incompleteness-corrected fractional luminosity function (FLF), namely, the number of star-forming regions per galaxy that contribute a given fraction of the total UV light of the galaxies. In Section 5, we compare the FLF of redshifted nearby galaxies with that of real galaxies. This comparison allows us to define "clumps" as star-forming regions whose fractional luminosity (FL) is significantly higher than that of redshifted nearby star-forming regions. Given this definition, we measure $f_{\text {clumpy }}$ in Section 6 and the clump contribution to the UV light and SFR of SFGs in Section 7. Conclusions and discussions will be presented in Section 8 .

Throughout the paper, we adopt a flat $\Lambda \mathrm{CDM}$ cosmology with $\Omega_{m}=0.3, \Omega_{\Lambda}=0.7$ and use the Hubble constant in terms of $h \equiv H_{0} / 100 \mathrm{~km} \mathrm{~s}^{-1} \mathrm{Mpc}^{-1}=0.70$. All magnitudes in the paper are in AB scale (Oke 1974) unless otherwise noted.

\section{DATA AND SAMPLE SELECTION}

\subsection{Catalogs and Images}

The sample of galaxies used in this paper is selected from the CANDELS/GOODS-S and UDS fields (Grogin et al. 2011; Koekemoer et al. 2011). CANDELS (HST-GO-12060) has observed both fields with the HST/WFC3 F160W band, reaching a $5 \sigma$ limiting depth (within a 0 '.17-radius aperture) of $27.36,28.16$, and $27.35 \mathrm{AB}$ mag for the GOODS-S wide ( $\sim 1 / 3$ of the GOODS-S), deep ( $\sim 1 / 3$ of the GOODS-S), and UDS fields. The remaining $1 / 3$ of the GOODS-S field has the F160W observation from ERS with a depth similar to that of the GOODS-S/deep region. Based on the source detection in the F160W band, the CANDELS team has made a multiwavelength catalog for each field, combining the newly obtained CANDELS HST/WFC3 data with existing public ground-based and space-based data. The details of the catalogs are given by Guo et al. (2013, for GOODS-S) and Galametz et al. (2013, for UDS). In brief, Hubble Space Telescope (HST) photometry was measured by running SExtractor on the point spread function (PSF)-matched images in the dual-image mode, with the F160W image as the detection image. Photometry in ground-based and IRAC images, whose resolutions are much lower than that of the F160W images, was measured by using TFIT (Laidler et al. 2007), which fit the PSF-smoothed high-resolution image templates to the low-resolution images to measure the fluxes in the low-resolution images.

Clumps are detected from the HST/ACS images of the galaxies. The spatial resolution of the Advanced Camera for 
Surveys (ACS) images $\left(0^{\prime} \cdot 1-00^{\prime} \cdot 12\right)$ is equivalent to $\sim 1 \mathrm{kpc}$ in our target redshift range. In GOODS-S, the images are the latest mosaics of the HST/ACS F435W, F606W, and F775W bands from the GOODS Treasury Program. They consist of data acquired prior to the HST Servicing Mission 4, including mainly data from the original GOODS $H S T /$ ACS program in HST Cycle 11 (GO 9425 and 9583; see Giavalisco et al. 2004) and additional data acquired on the GOODS fields during the search for high redshift Type Ia supernovae carried out during Cycles 12 and 13 (Program ID 9727, PI: Saul Perlmutter, and 9728, 10339, 10340, PI: Adam Riess; see, e.g., Riess et al. 2007). The $5 \sigma$ limiting depths (within a 0 '.17-radius aperture) of ACS F435W, F606W, and F775W bands in the GOODS-S field are 28.95, 29.35, and 28.55 AB, respectively. In UDS, CANDELS has taken parallel observations on the F606W and F814W bands, with the $5 \sigma$ limiting depths of 28.49 and $28.53 \mathrm{AB}$.

Besides doubling our sample size, using both the GOODS-S and UDS fields allows us to evaluate the incompleteness of clump detections at different observation depths.

\subsection{Galaxy Properties}

The properties of galaxies in the two fields are measured through fitting the broad-band spectral energy distributions (SED) in the catalogs to synthetic stellar population models. We use the official CANDELS photometric redshift (photo- $z$ ) catalogs in the two fields, which combine the results from more than a dozen photo- $z$ measurements with various SED-fitting codes and templates. The technique is fully described in Dahlen et al. (2013). Stellar mass and other stellar population properties (such as age, extinction, UV-based SFR, etc.) are measured by using FAST (Kriek et al. 2009), with redshift fixed to the best available ones (spectroscopic or photometric). The modeling is based on a grid of Bruzual \& Charlot (2003) models that assume a Chabrier (2003) initial mass function, solar metallicity, exponentially declining star formation histories, and a Calzetti extinction law (Calzetti et al. 1994, 2000).

SFRs are measured on a galaxy-by-galaxy basis using a ladder of SFR indicators as described in Wuyts et al. (2011). The method essentially relies on IR-based SFR estimates for galaxies detected at mid- to far-IR wavelengths, and SEDmodeled SFRs for the rest. As shown in Wuyts et al. (2011) the agreement between the two estimates for galaxies with a moderate extinction (faint IR fluxes) ensures the continuity between the different SFR estimates. For IR-detected galaxies the total SFRs, SFR IR+UV, were then computed from a combination of IR and rest-frame UV luminosity (uncorrected for extinction) following Kennicutt (1998). We refer readers to Barro et al. (2011, 2013) for the details of our measurements of galaxy properties.

\subsection{Sample}

We select SFGs with $M_{*}>10^{9} M_{\odot}$, SSFR $>10^{-1} \mathrm{Gyr}^{-1}$, and $0.5<z<3$ in both fields to study $f_{\text {clumpy. To ensure }}$ a clean source detection with small photometric uncertainty in the F160W band, we also require all galaxies to have $H_{\text {F160w }}<24.5 \mathrm{AB}$. This apparent magnitude cut only affects the mass completeness of our sample at $z>2$. As shown in the lower panel of Figure 1, a typical SFG (for example, a constant star-forming model with age of $0.5 \mathrm{Gyr}$ ) with dust extinction $E(B-V)=0.15$ and $\log \left(M_{*} / M_{\odot}\right)>9.0$ has an apparent $\mathrm{F} 160 \mathrm{~W}$ magnitude brighter than $24.5 \mathrm{AB}$ at $0.5<z<2$. At $z>2$, the mass completeness limit under this apparent magnitude cut increases with redshift and reaches

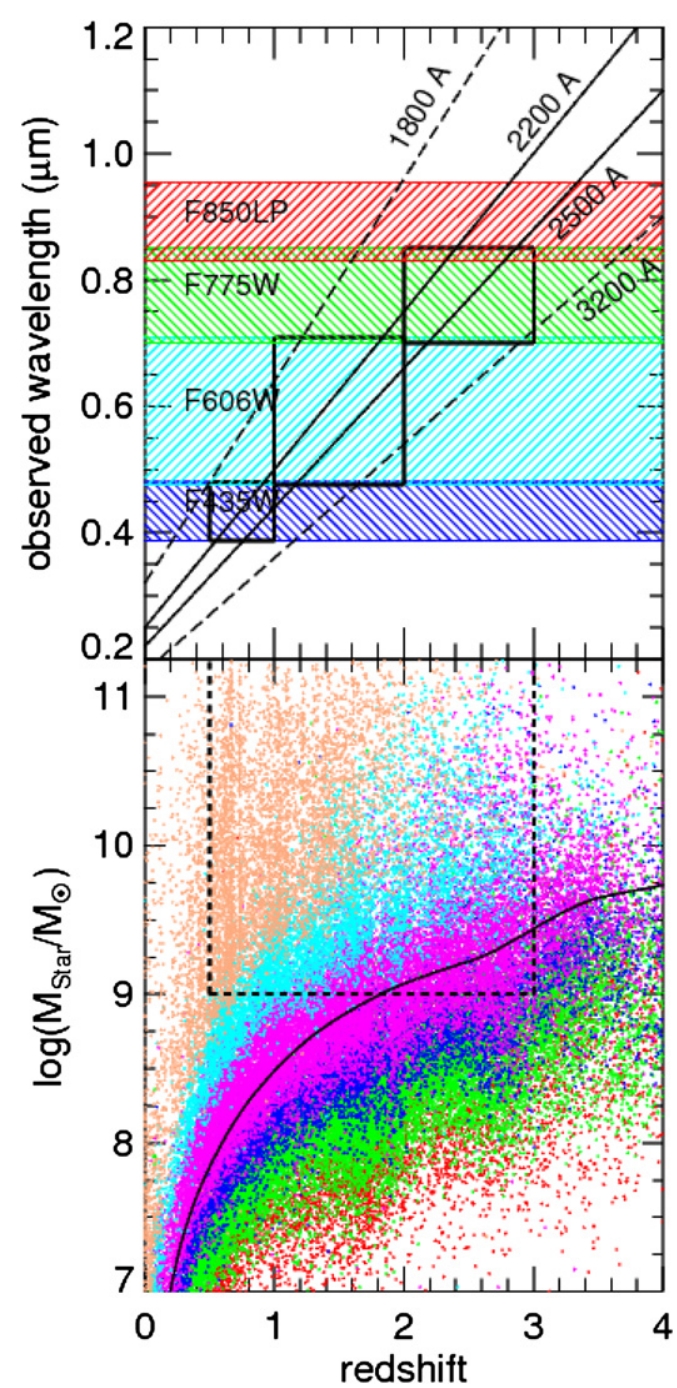

Figure 1. Upper: shift of our clump detection bands. The colored areas show the wavelength coverage of ACS filters. The large black rectangles show the bandpass used for detecting clumps in each redshift range in our study. Black lines, from bottom to top, show the observed wavelength of rest-frame 1800 , 2200, 2500, and $3200 \AA$, respectively. Lower: $M_{*}$-redshift diagram of the CANDELS/GOODS-S catalog. Galaxies with $H_{\mathrm{F} 160 \mathrm{~W}} \geqslant 27.0$ (red), $26.0 \leqslant$ $H_{\text {F160w }}<27.0$ (green), $25.0 \leqslant H_{\text {F160w }}<26.0$ (blue), $24.0 \leqslant H_{\text {F160w }}<25.0$ (purple), $23.0 \leqslant H_{\mathrm{F} 160 \mathrm{~W}}<24.0$ (cyan), and $H_{\mathrm{F} 160 \mathrm{~W}} \leqslant 23.0$ (light brown) are shown. The black curve shows the $M_{*}$ of an SED template with $H_{\mathrm{F} 160 \mathrm{~W}}=24.5$ $\mathrm{AB}$ and a constant star formation history over an age of $0.5 \mathrm{Gyr}$ at different redshifts. Black dashed lines show the boundary of our sample.

$\log \left(M_{*} / M_{\odot}\right) \sim 9.4$ at $z=3$. In our later analyses, we still include galaxies with $M_{*}$ down to $\log \left(M_{*} / M_{\odot}\right)=9.0$ at $z>2$, but remind readers that our lowest $M_{*}$ bin at $z>2$ is incomplete because of the apparent magnitude cut.

The apparent magnitude cut of $H_{\mathrm{F} 160 \mathrm{w}}<24.5 \mathrm{AB}$ also ensures us a reliable morphology and size measurements of our galaxies. In our study, the size (semi-major axis, $r_{e}$ or SMA hereafter) and axial ratio $(q)$ of each galaxy are taken from van der Wel et al. (2012), who measured these parameters by running GALFIT (Peng et al. 2002) on the CANDELS F160W images. van der Wel et al. (2012) showed that the random uncertainty of both $r_{e}$ and $q$ is $\sim 20 \%$ at $H_{\mathrm{F} 160 \mathrm{~W}}=24.5 \mathrm{AB}$, and quickly increases to about $\sim 50 \%$ at $H_{\mathrm{F} 160 \mathrm{~W}}=25.5 \mathrm{AB}$. The $\mathrm{SFR}-M_{*}$ and size (SMA) $-M_{*}$ relations of our sample are shown in Figure 2.

We also exclude galaxies whose sizes are less than $0^{\prime \prime} .2$, because clumps cannot be resolved in these marginally resolved 


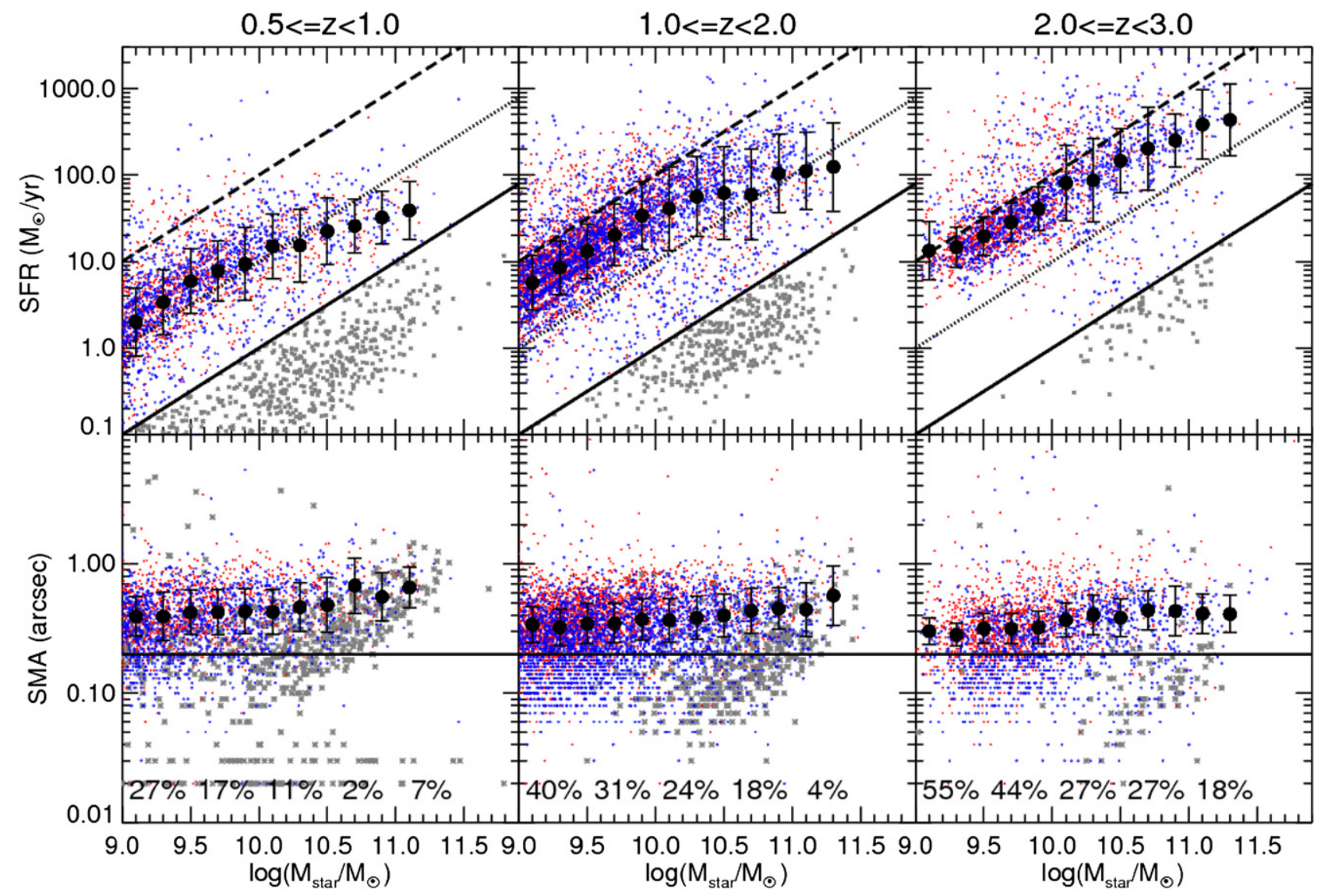

Figure 2. Sample selection. Galaxies in the CANDELS/GOODS-S and UDS with $H_{\mathrm{F} 160 \mathrm{~W}}<24.5$ AB are plotted in the SFR-M* and semi-major axis (SMA) $-M_{*}$ diagrams. Galaxies with SSFR $>0.1 \mathrm{Gyr}^{-1}$ and axial ratio $q>0.5$ (blue) are selected into our sample of clump detection. Red points show galaxies with SSFR $>0.1 \mathrm{Gyr}^{-1}$ but $q \leqslant 0.5$, while gray points show galaxies with SSFR $\leqslant 0.1 \mathrm{Gyr}^{-1}$. Black filled circles with error bars show the median and scatter of the star-forming galaxies (with SSFR $>0.1 \mathrm{Gyr}^{-1}$ ) in the SFR- $M_{*}$ diagram. Black solid, dotted, and dashed lines in the upper panels show the relations of SSFR $=$ $0.1,1$, and $10 \mathrm{Gyr}^{-1}$. Black horizontal lines in the lower panels show our size cut of 0 '.2. Blue points below the size cut are excluded from our sample. The fraction of the blue points that are excluded due to the small sizes is labeled in the lower panels for each $M_{*}$ bin (starting from $\log \left(M_{*} / M_{\odot}\right)=9$ and increasing with a width of $0.5 \mathrm{dex})$.

or unresolved sources. In the lower panel of Figure 2, we give the fraction of the galaxies that are excluded because of their small size in each $M_{*}$ and redshift bin. If we assume that galaxies in each (redshift, $M_{*}$ ) bin are self-similar despite their different sizes, $f_{\text {clumpy }}$ and the clump contribution measured from the resolved galaxies in later sections are still representative for the whole SFG population in the (redshift, $M_{*}$ ) bin. On the other hand, if we believe that there are physical reasons that make the unresolved galaxies non-clumpy, we should scale down our the $f_{\text {clumpy }}$ and the clump contribution in our later analyses by the fraction of the unresolved galaxies in each (redshift, $M_{*}$ ) bin.

The above two assumptions are two extremes that our $f_{\text {clumpy }}$ can be easily used to infer the $f_{\text {clumpy }}$ of all SFGs regardless of their sizes. The real situation, however, could be in between the two extremes. For example, smaller (unresolved) galaxies may have intrinsically fewer clumps and lower $f_{\text {clumpy }}$. In this case, the $f_{\text {clumpy }}$ of unresolved galaxies cannot be simply inferred from the $f_{\text {clumpy }}$ of resolved galaxies. If that is true, current data cannot address the $f_{\text {clumpy }}$ of unresolved galaxies, observations with higher spatial resolutions are needed.

To minimize the effect of dust extinction and clump blending, we only use galaxies with axial ratio $q>0.5$. This $q$ criterion excludes some very elongated clumpy galaxies, such as chain galaxies in Elmegreen \& Elmegreen (2005) and Elmegreen et al. (2007). As shown by Elmegreen \& Elmegreen
(2005), the axial ratio distribution of chain galaxies plus clumpclusters is constant, as expected for randomly oriented disks. Ravindranath et al. (2006), however, found that the axial ratio distribution of high-redshift Lyman Break Galaxies is skewed toward the high-value end, against the scenario of randomly oriented disks. Although the galaxy number distribution in our sample is skewed toward lower $q$ in the high-redshift low-mass range (i.e., more red points than blue points above the size cut at $\log \left(M_{*} / M_{\odot}\right)<10$ in the lower right panel of Figure 2), galaxies with $q>0.5$ (blue points) and $q \leqslant 0.5$ (red points) follow almost the same SFR- $M_{*}$ and SMA- $M_{*}$ relations in Figure 2. Therefore, we believe that, in general, the properties of the clumps in $q>0.5$ galaxies are likely to be representative of those in all SFGs, regardless of their inclinations. Furthermore, excluding very elongated galaxies reduces the rate of problematic detections by our clump finder, which tends to overdeblend elongated galaxies.

After the above selection criteria, and further excluding galaxies that are not covered by the ACS images, the final sample consists of 3239 galaxies.

\section{DETECTING DISCRETE STAR-FORMING REGIONS}

We begin our clump definition by searching for clumps among discrete star-forming regions, believing that clumps occupy the bright end of the luminosity distribution of the discrete 


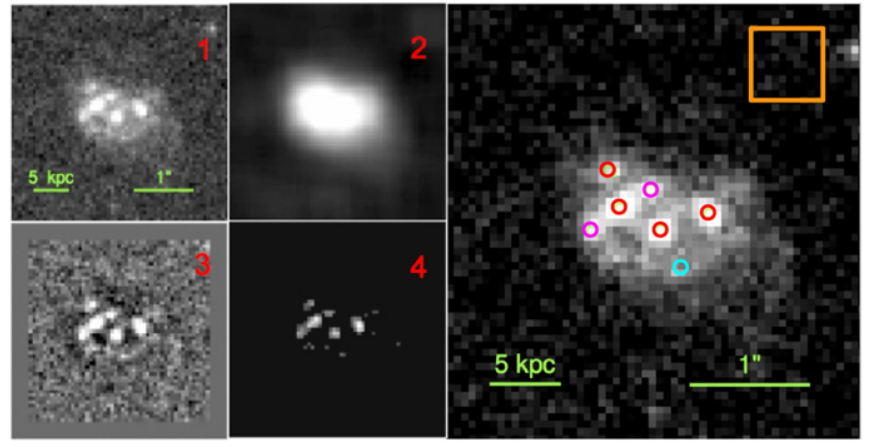

Figure 3. Illustration of the process of our blob finder. First, the original image (panel 1) is smoothed. The smoothed image (panel 2) is then subtracted from the original image to make a contrast image (panel 3). After low-S/N pixels are masked out, blobs are detected from the filtered image (panel 4). The final detected blobs (red and magenta) are shown in the right panel. The orange box in the right panel shows the size of the smoothing box $\left(0^{\prime \prime} 6\right)$. The blob detection depends on the size of the smoothing box. If the box size is reduced by half to 0 .' 3 , only red blobs are detected. And if the box size is doubled to $1 . .2$, a new blob (cyan) will be added to the detection.

star-forming regions. Before separating clumps from ordinary star-forming regions (i.e., individual or blended $\mathrm{H}$ II regions), we call all regions detected in this section "blobs" for simplicity.

\subsection{Automated Star-forming Region Finder}

We design an automated blob finder to detect blobs from the galaxies in our sample. The process of the blob finder is illustrated in Figure 3. We first cut a postage stamp for each galaxy from its clump detection image. The size of the postage stamp is determined by the dilated segmentation area of the source. The process of "dilation" extends the SExtractor F160W segmentation area generated in our source detection (Guo et al. 2013; Galametz et al. 2013) to a proper size to include the outer wing of the object below the SExtractor isophotal detection threshold (see Galametz et al. 2013, for details). We then smooth the postage stamp (Panel 1) through a boxcar filter with size of 10 pixels $\left(00^{\prime} 6\right)$ to obtain a smoothed image (Panel 2). Then, we subtract the smoothed image from the original image to make a contrast image (Panel 3). The above steps are similar to those used in calculating the "Clumpiness (S)" of the CAS system of Conselice (2003). We then measure the background fluctuation from the contrast image after $3 \sigma$-clipping. We then mask out (set value to 0 ) all pixels below $2 \sigma$ of the background fluctuation to make a filtered image (Panel 4), where blobs stand out in a zero background. We then run SExtractor on the filtered image to detect sources, requiring a minimal detection area of 5 pixels to exclude spurious detections. Each detected source is considered as one blob. In the example of Figure 3, the detected blobs are shown by red symbols in the right panel. A comparison with the CANDELS visual clumpiness (Kartaltepe et al. 2014) shows our automated finder works well for identifying discrete star-forming regions (see Appendix).

The blob detection depends on the size of the smoothing box. Our choice of 10 pixels (0.' 6$)$, shown by the orange box in the right panel of Figure 3, is the optimized one according to our later test of fake blobs (Section 3.3) and comparison with the CANDELS visual inspection (Appendix). As long as the smoothing length is significantly larger than the typical size of blobs, blobs would stand out in the contrast image (panel 3 of Figure 3) and hence be detected. Since the smoothing length of 0 .' $6(\sim 5 \mathrm{kpc}$ at $0.5<z<3)$ is significantly larger than the typical size of blobs $(<1 \mathrm{kpc})$, most of the UV-bright blobs should be able to stand out in the contrast image unless their sizes are close to $5 \mathrm{kpc}$. If, however, the smoothing length is too large, some noisy pixels may also be able to stand out in the contrast image and hence be detected as a blob. We demonstrate the effect of using different smoothing lengths in Figure 3. If we use 5 pixels ( 0.3 ) to smooth the image, two obvious blobs (magenta) would be missed. On the other hand, if we use 20 pixels (1".2), a new blob (cyan) would be detected. This cyan blob, however, is likely a spurious detection and would be excluded by our later clump definition (Section 5). More examples of identified blobs in clumpy galaxies can be found in Figure 4.

\subsection{Detection and Measurement}

We detect blobs in different HST/ACS bands based on the redshift of the galaxies. The choice of the detection filter in GOODS-S is shown in the upper panel of Figure 1: F435W for galaxies at $0.5<z<1.0, \mathrm{~F} 606 \mathrm{~W}$ for galaxies at $1.0<z<2.0$, and F775W for galaxies at $2.0<z<3.0$. The purpose of the choice is to detect blobs in the same rest-frame UV range, namely $2000 \AA-2800 \AA$, at different redshifts. For UDS, we use $\mathrm{F} 814 \mathrm{~W}$ to replace the $\mathrm{F} 775 \mathrm{~W}$ for galaxies at $2.0<z<3.0$. There are, however, no HST observations close to $\mathrm{F} 435 \mathrm{~W}$ available in UDS. As a compromise, we use F606W to detect blobs for galaxies at $0.5<z<1.0$ in UDS. We will discuss the systematic offsets introduced by this band mismatch later.

Once a blob has been detected, we measure its flux in the detection band by assuming it is a point source. The assumption is validated by the statistics of the light profile of all detected blobs in Figure 5. The average light profile of blobs with given fractional luminosity ( $\mathrm{FL}=L_{\text {blob }} / L_{\text {galaxy }}$ ) in a given redshift and host galaxy mass bin is very well described by the light profile of the PSF of the detection band plus the average background of the blobs. Here we assume the light profile at $r>6$ pixel (0.36) is dominated by the background ("disk" component) light. The only exception happens for faint blobs $(\mathrm{FL}<0.1)$ in the lowest redshift bin $(0.5<z<1.0)$, where the average blob profile is broader than that of the PSF. The PSF profile, however, still lies within the $1 \sigma$ range of the blob profiles, implying that the blobs are only marginally resolved. Overall, we conclude that the detected blobs are just marginally, if at all, resolved and the assumption of a point source would not introduce significant systemics in measuring the blob fluxes.

When measuring the flux of each blob, we first determine the background light from the azimuthally averaged flux at $r=$ 6-10 pixels away from the blob center, after masking out the central region ( $r<4$ pixels) of all other blobs. We then extrapolate the background flux to the center of the blob. After subtracting the background, we measure an aperture flux with radius $r=$ 3 pixels. This background-subtracted aperture flux is finally scaled up based on the curve-of-growth of the corresponding PSF to obtain the total flux of the point-like blobs.

We choose to subtract the local background of blobs, because we believe that the blobs are "embedded" in the galaxies. Whether or not the local background should be subtracted is still an open issue in clump studies (e.g., Förster Schreiber et al. 2011; Guo et al. 2012; Wuyts et al. 2012). In fact, the background subtraction is also a controversial issue for studying local star-forming regions. It even affects our understanding of the basic physics of star formation, e.g., the slope of the Kennicutt-Schmidt Law (see the comparison between Bigiel et al. (2008) and Liu et al. (2011)). If we do not subtract the local background and scale up the total aperture flux within $r=3$ pixels according to the PSF profile, the fluxes of our blobs will be systematically higher by a factor of two. 

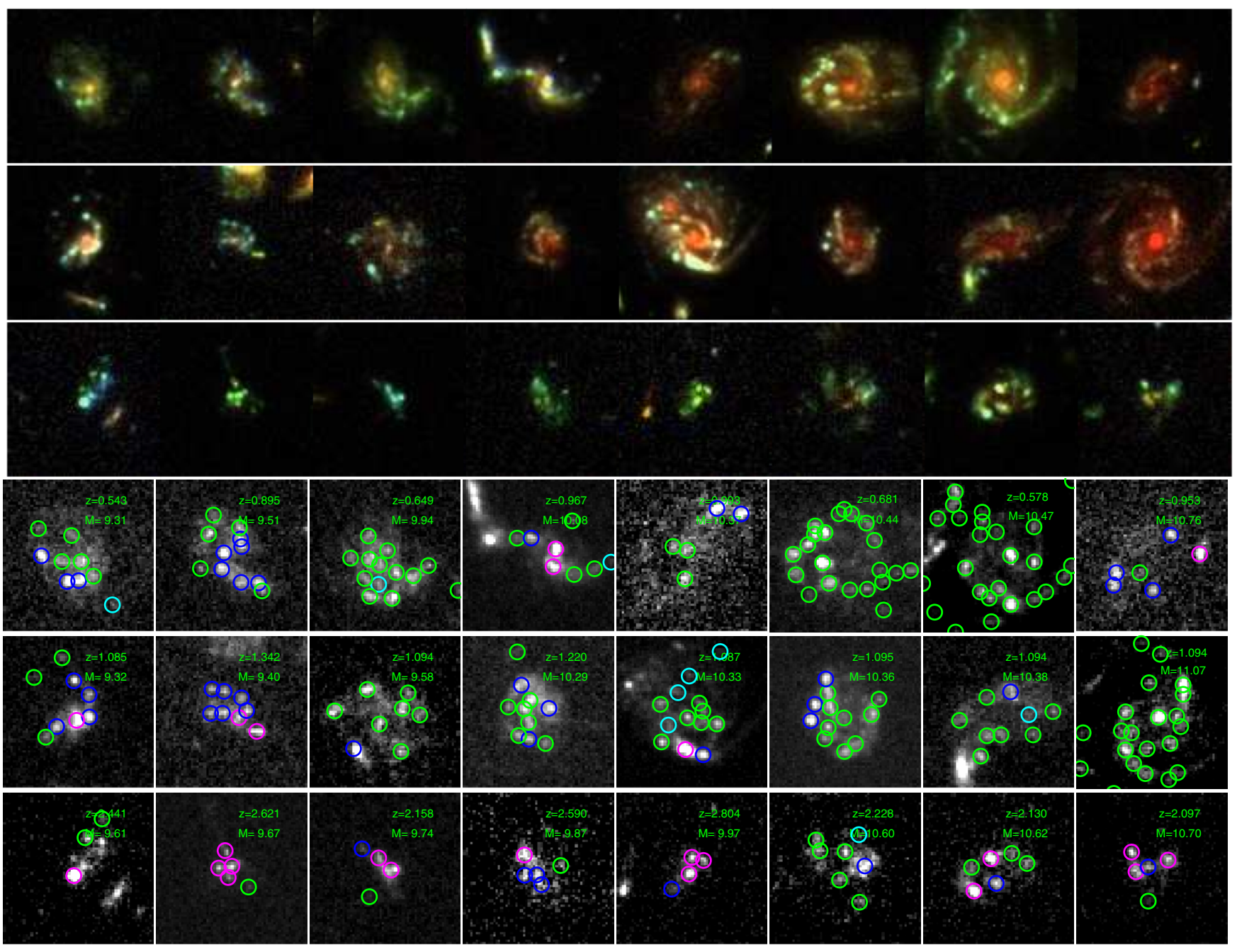

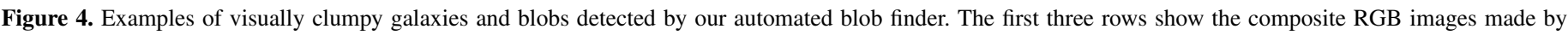

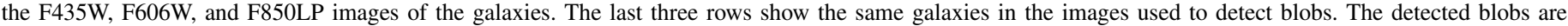

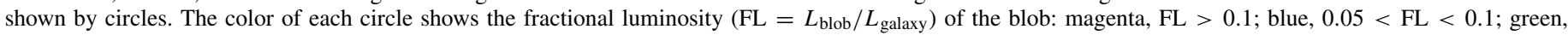

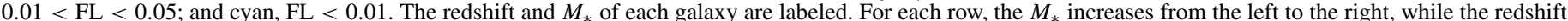

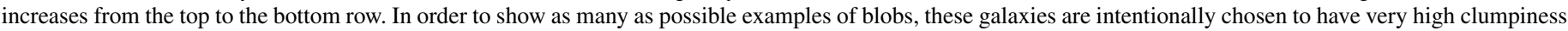

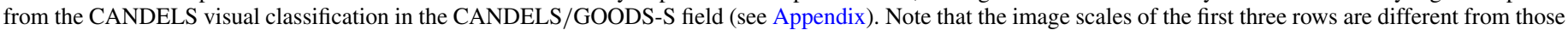
of the last three rows.

\subsection{Completeness of the Blob Finder}

We evaluate the completeness of our blob finder by recovering fake blobs. For each galaxy in our sample, regardless of whether it contains detected blobs, we insert one fake blob into its image in the detection band and re-run our blob finder on it. We use point sources to mimic the blobs. This simplification is validated by the fact that the light profile of blobs can be well described by the PSF of the detection bands (Figure 5). The fluxes of fake blobs are randomly selected from a uniform distribution between $1 \%$ and $20 \%$ of the flux of their galaxies. The fake blobs are only added into the segmentation areas of the galaxies. For each galaxy, we repeat the process 30 times to improve the statistics. Comparing with the method of adding arbitrary numbers of blobs to fake model galaxies (e.g., Sérsic models), our method largely preserves the distributions of the size, magnitude, surface brightness profile, and blob crowdedness of real galaxies, which are all important to the blob detection probability.

The detection probability, i.e., the successful rate of recovering fake blobs, depends on the properties of both galaxies and blobs. More specifically, it depends on redshift $(z)$, the magnitude of galaxies $\left(\operatorname{mag}_{g}\right)$, the size of galaxies $\left(r_{e}\right)$, the magnitude of blobs $\left(\mathrm{mag}_{b}\right)$, the location of blobs (the distance to the center of the galaxies, $d_{b}$ ), and the number of blobs in the galaxies $\left(n_{b}\right)$. For each of the real blobs, we assign a detection probability to it based on its values of the above parameters, $P\left(z, \operatorname{mag}_{g}, r_{e}, \operatorname{mag}_{b}, d_{b}, n_{b}\right)$, if we have at least five detected fake blobs in the $\left(z, \operatorname{mag}_{g}, r_{e}, \operatorname{mag}_{b}, d_{b}, n_{b}\right)$ bin. Otherwise, we determine its probability by interpolating the marginalized detection probability as a function of the FL of the blobs (the second row of Figure 6). In fact, using the probability $-\operatorname{mag}_{b}$ relation (the first row of Figure 6) also provides a good approximation for blobs in the under-sampled bins, but using the probability-FL relation makes our later analyses easy because we are measuring the FLF instead of the absolute luminosity function. Only $\lesssim 10 \%$ of our blobs fall in the under-sampled $\left(z, \operatorname{mag}_{g}, r_{e}, \operatorname{mag}_{b}, \widetilde{d}_{b}, n_{b}\right)$ bins. Using the interpolated marginalized detection probability would not affect our later results.

In order to avoid possible contamination from bulges, which usually stand out in the filtered images (Panel 3 of Figure 3 and hence almost always are detected as blobs, we also exclude blobs that are within $d_{b}<0.5 \times r_{e}$. For example, we only count 

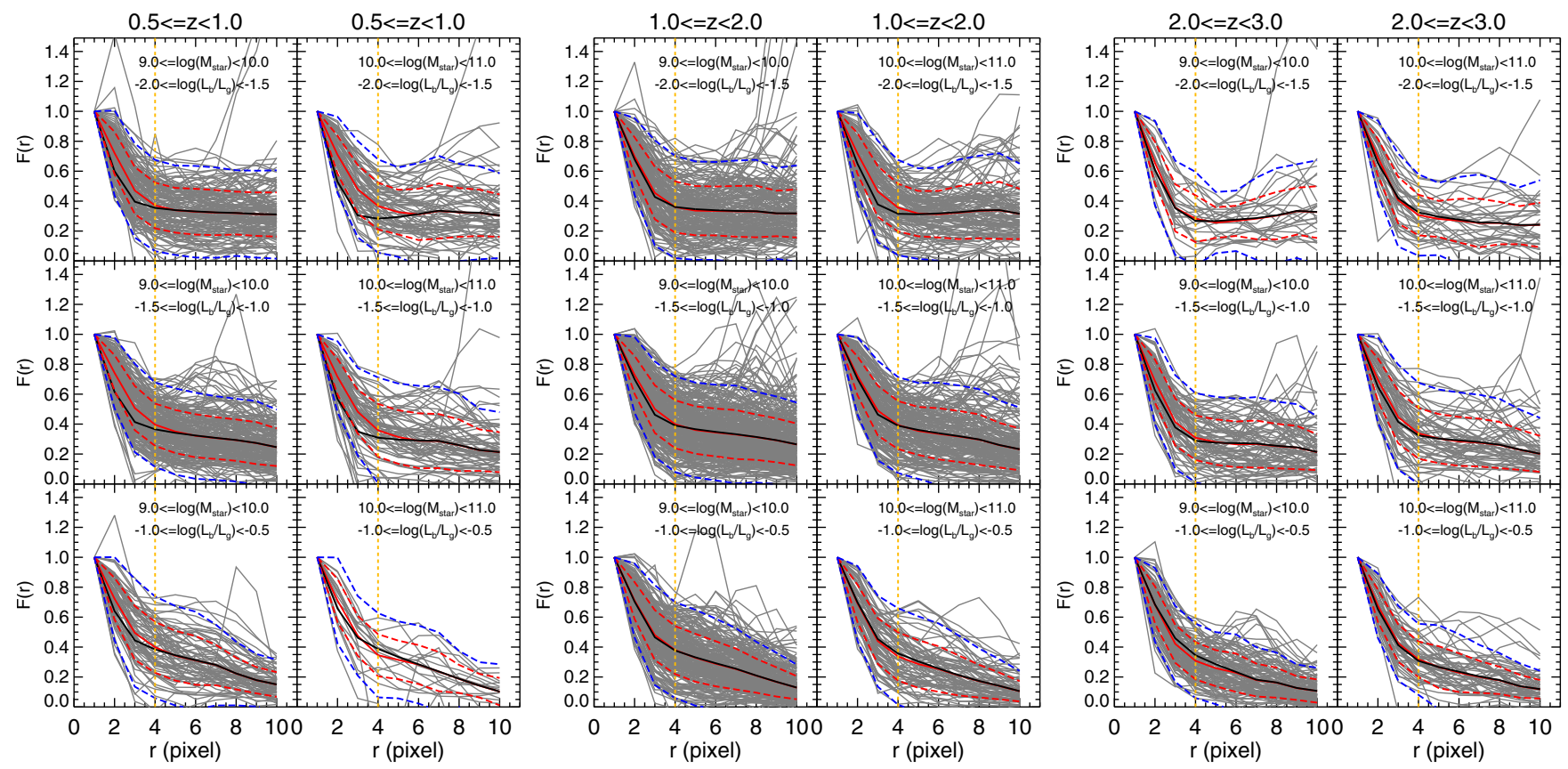

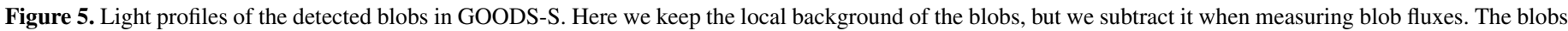

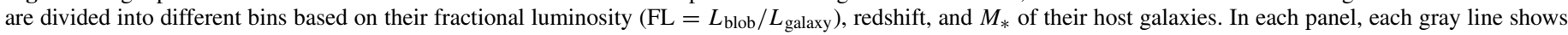

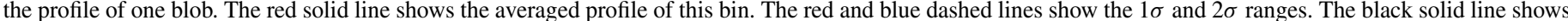

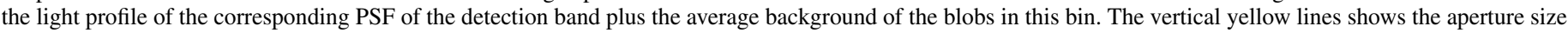
used to measure the blob fluxes.

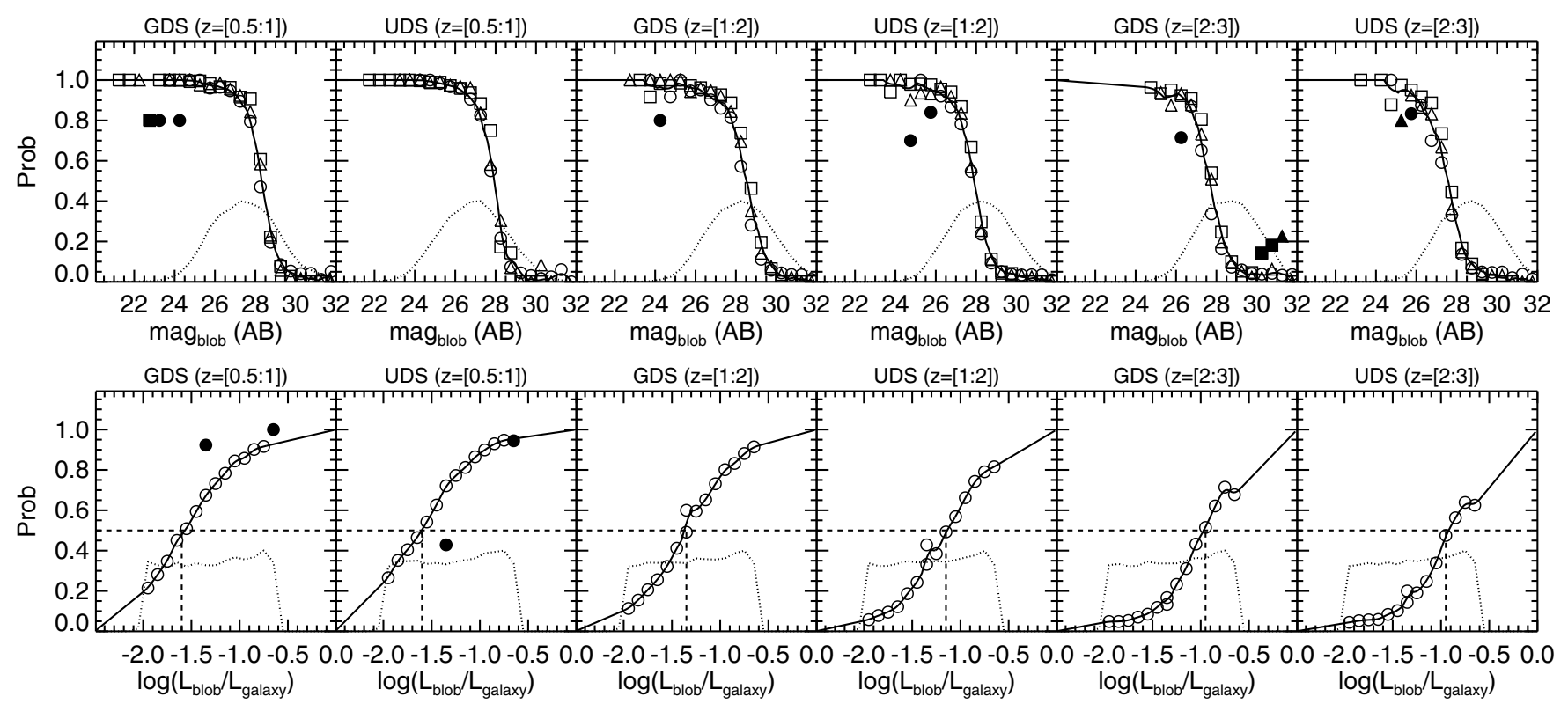

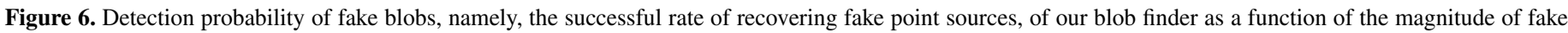

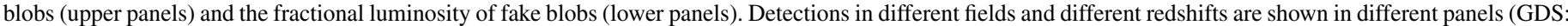

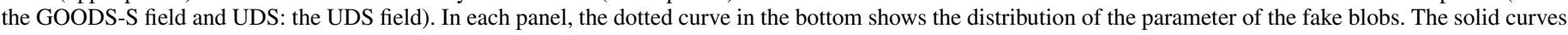

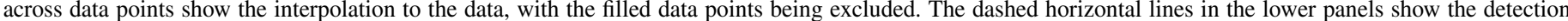
probability of $50 \%$, while the dashed vertical lines show the corresponding fractional luminosity of the $50 \%$ detection probability.

five blobs in the galaxy in Figure 3. We also exclude blobs that are beyond $d_{b}>8 \times r_{e}$ (if the size of the postage stamp of a galaxy is larger than $8 \times r_{e}$ ), in order to reduce the impact of nearby small satellite galaxies.

We also measure the fluxes of the fake blobs using the method described in Section 3.2 and compare them with the input values. In general, the measured and input values show good agreement. There is, however, a mild trend that the fluxes are overestimated as the galactocentric distance of the blobs $\left(d_{b}\right)$ decreases, with the maximum overestimation of $\sim 30 \%$ for blobs at $0.5 \times r_{e}$. We fit the overestimation $-d_{b}$ relation and scale down the flux of each real blob based on its $d_{b}$.

\section{FRACTIONAL LUMINOSITY FUNCTION OF BLOBS}

Now, we measure the FLF of the blobs, taking into account the detection incompleteness. Since our fake blobs only have the 


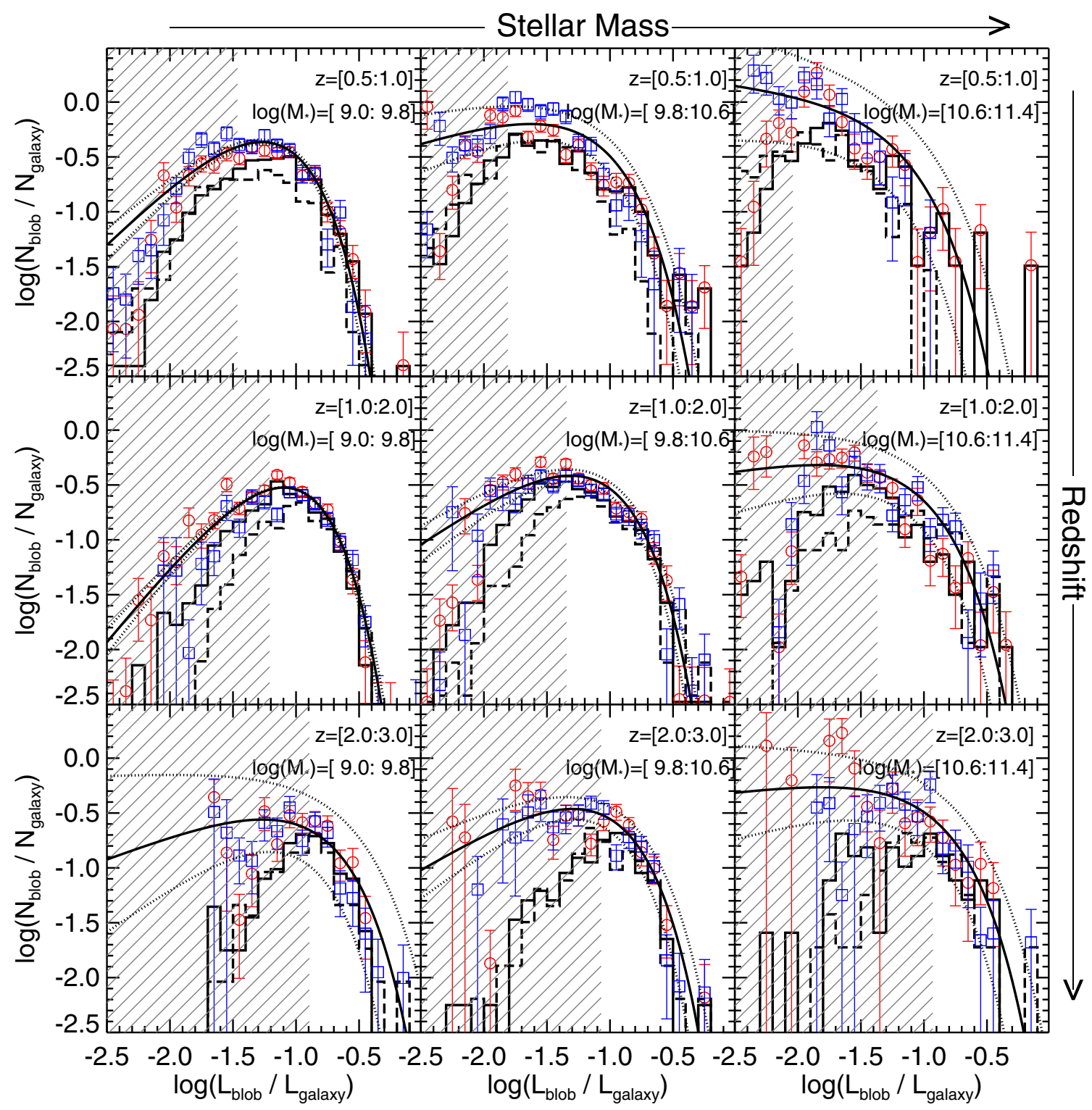

Figure 7. Fractional luminosity functions of blobs. Each panel shows the average number of blobs per galaxy, as a function of the fractional luminosity of the blobs, in galaxies within a given redshift and $M_{*}$ bin. Solid (GOODS-S) and dashed (UDS) histograms show the results without being corrected for the blob detection incompleteness. Red (GOODS-S) and blue (UDS) symbols show the results after the incompleteness correction. Error bars are derived from the Poisson error of the blob number counts. The shaded area in each panel shows the region where the blob detection incompleteness is larger than 50\% (see the dashed vertical lines in the second row of Figure 6 for an example of how the 50\% threshold is determined, but note that each panel of Figure 6 includes galaxies with all $M_{*}$, while galaxies are separated into different $M_{*}$ bins in this figure). The solid and dashed black curves in each panel show the best-fit Schechter Function and its confidence interval for the combined GOODS-S and UDS fractional luminosity functions.

fractional fluxes down to $L_{\text {blob }} / L_{\text {galaxy }}=0.01$, we extrapolate the detection probability for fainter blobs using their fractional luminosity. It is important to note that the incompleteness estimated in Section 3.3 only tells us the fraction of blobs that are missed by our blob finder. It does not tell us from which galaxies, "blobby" or "non-blobby," they are missed. Some "non-blobby" galaxies may actually contain a few blobs, which are somehow missed in our detection. Since these missed blobs are taken into account in the incompleteness, their host galaxies, which are misclassified as "non-blobby," should also be taken into account when we measure the FLF. Therefore, our FLF and later UV light and SFR contributions from blobs (or clumps) are measured for all SFGs rather than just for the galaxies with detected blobs (or clumps).

The FLFs of the GOODS-S and UDS fields, both before and after the incompleteness corrections, are shown in Figure 7. The results at $1<z<2$ are very encouraging, demonstrating that our fake blob test correctly evaluates the incompleteness of our blob detection. In this redshift bin, both GOODS-S and UDS fields select blobs from the HST F606W band, but the depths of their F606W images are different. The GOODS-S image is about two times deeper (in terms of exposure time) than the UDS one. As a result, the uncorrected FLF (black histograms in the figure) of GOODS-S is about 1.5-2 times higher than that of UDS for blobs with $L_{\text {blob }} / L_{\text {galaxy }}<0.1$. After correcting the incompleteness, both functions (symbols with error bars) of GOODS-S and UDS show excellent agreement in all three $M_{*}$ ranges. This result indicates that after the correction, our results are largely unaffected by the varying observation depth from field to field.

At $0.5<z<1$, due to the lack of $\mathrm{F} 435 \mathrm{~W}$ images in the UDS field, we must use the CANDELS parallel F606W 

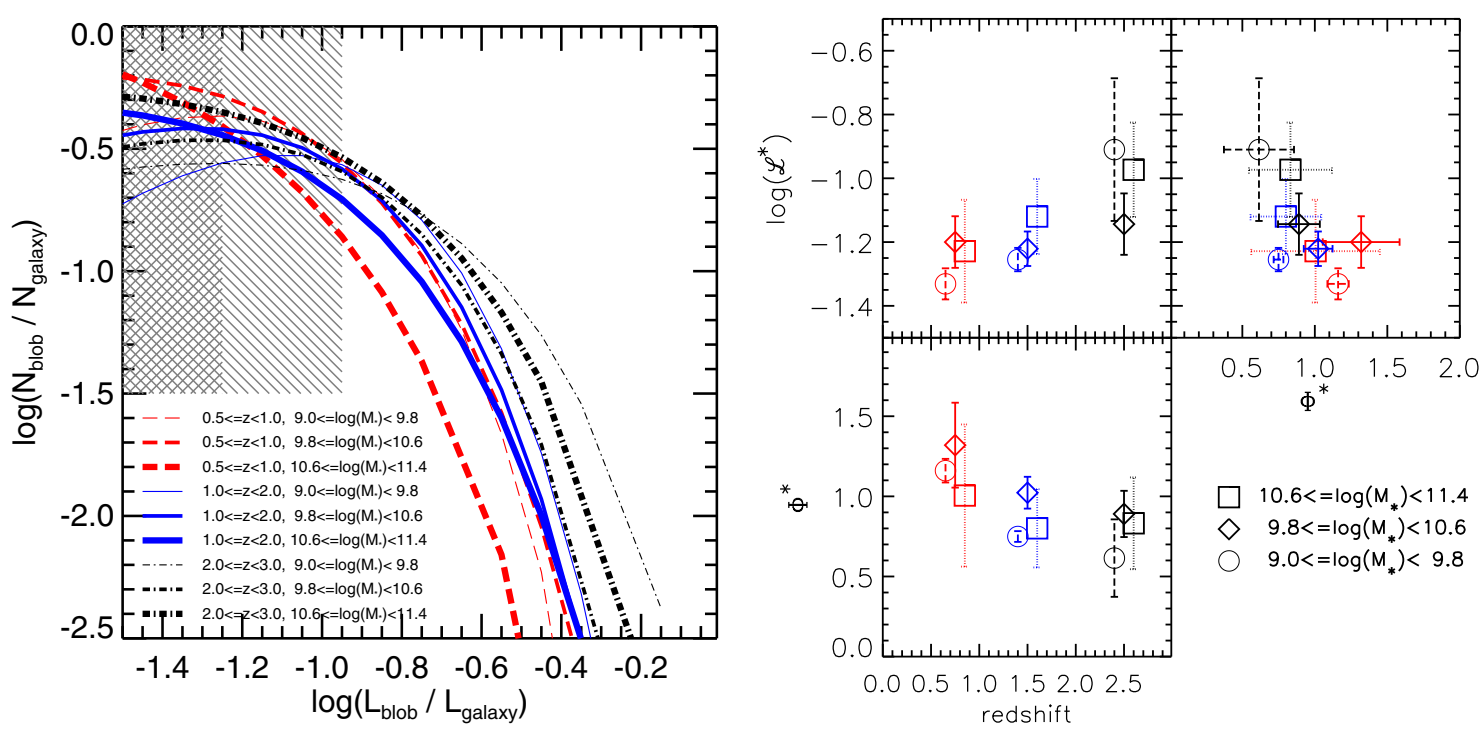

Figure 8. Left: best-fit Schechter functions to the fractional luminosity functions of blobs in all redshift and galaxy $M_{*}$ bins. The shaded area shows the region where the blob detection incompleteness is larger than $50 \%$ for galaxies at $2<z<3$, while the double-shaded area shows the same region at $1<z<2$. The 50\% incomplete region for galaxies at $0.5<z<1$ is about $\log \left(L_{\mathrm{blob}} / L_{\text {galaxy }}\right)=-1.5$. Right: best-fit $\Phi^{*}$ and $\mathscr{L}^{*}$ of the Schechter functions of all redshift and galaxy $M_{*}$ bins. Red, blue, and black symbols are for galaxies at $0.5<z<1,1<z<2$, and $2<z<3$, respectively.

image to detect blobs. At this redshift range, F606W samples the rest-frame $U$-band, while $\mathrm{F} 435 \mathrm{~W}$ samples the rest-frame $2500 \AA$. As found by Wuyts et al. (2012), the UV luminosity contribution of blobs decreases as the detection bands shift from blue to red. If we extrapolate the UV luminosity contribution of star-forming regions detected in different bands of Wuyts et al. (2012) to the rest-frame $2500 \AA$, the difference of blob luminosity contribution between $2500 \AA$ and $U$-band is about a factor of 1.7. After being scaled up, the UDS FLF matches the GOODS-S FLF very well at $0.5<z<1$ in all $M_{*}$ bins (the top panels of Figure 7).

In the highest redshift bin, $2<z<3$, the incompleteness corrected results of the two fields also show agreement, but with larger uncertainties for faint blobs $\left(L_{\text {blob }} / L_{\text {galaxy }}<0.03\right)$, which are hard to detect at such high redshifts. With a very small number of detected faint blobs, our incompleteness correction method has difficulty to properly recover the real blob numbers. We note that, however, our later analyses use little information from these high-redshift faint blobs.

It is important to note that the faint end of each incompleteness-corrected FLF in Figure 7 should be treated with caution. In most panels, the FLF decreases in the faint end, suggesting that the incompleteness is somehow not properly corrected in the faint end, although our correction method shows encouraging results in the bright and intermediate regions. Some small and faint blobs would be missed by our blob finder, because their sizes do not satisfy our minimal area requirement of 5 pixels. Such blobs may not be properly taken into account in our fake blob simulations, which results in an underestimate of the incompleteness.

In Figure 7, we shadow the regions where the incompleteness is larger than $50 \%$, i.e., the marginalized probability of detecting a fake blob as a function of the FL of blob is less than $50 \%$. The second row of Figure 6 shows an example of how this $50 \%$ threshold is determined. It should be noted that in Figure 6, we do not separate galaxies into different $M_{*}$ bins, but we do so in Figure 7. Therefore, the 50\% thresholds in Figure 7 are slightly different from those in Figure 6. Also, we use the average threshold of GOODS-S and UDS in each panel of Figure 7. In the shaded regions, the shape of the FLF depends on the accuracy of our incompleteness correction method more than on the number of detected blobs.

To study the evolution of the FLFs with redshift and $M_{*}$, we fit a Schechter Function (Schechter 1976) to each FLF in Figure 7:

$$
n(\mathscr{L}) d \mathscr{L}=\Phi^{*} \times\left(\mathscr{L} / \mathscr{L}^{*}\right)^{\alpha} \times e^{-\left(\mathscr{L} / \mathscr{L}^{*}\right)} d \mathscr{L},
$$

where $\mathscr{L}=L_{\text {blob }} / L_{\text {galaxy }}$. The best-fit functions and their parameters, $\Phi^{*}$ and $\mathscr{L}^{*}$, are shown in Figure 8 . We do not show $\alpha$ because it strongly depends on the very faint end of the fractional luminosity function (e.g., $L_{\text {blob }} / L_{\text {galaxy }} \sim 0.01$ ), where our blob detection completeness is very low (only 10\%-20\%). The dependences of $\Phi^{*}$ and $\mathscr{L}^{*}$ on the very faint end are weaker than that of $\alpha$. For the ranges of $0.5<z<1$ and $1<z<2$, we fit the functions down to the faint luminosity of $\log (\mathscr{L})=-2.5$, while in the highest redshift range, $2<z<3$, we only fit the functions down to $\log (\mathscr{L})=-2.0$ due to the large error bars and missing data in some luminosity bins (e.g., blobs around $L_{\text {blob }} / L_{\text {galaxy }} \sim-2.0$ in the least massive bin in this redshift in Figure 7). We also overplot the best-fit functions and their uncertainty ranges in Figure 7. It is important to note that the choice of the Schechter Function is empirical and not driven by any physical reasons. In fact, a truncated power-law is usually used to study the bright end of the luminosity functions of nearby H II regions (e.g., Scoville et al. 2001; Liu et al. 2013). We choose the Schechter Function because it fits both the bright and faint ends.

The trends of the best-fit Schechter parameters can be clearly seen from the right panels of Figure 8. For galaxies with a given $M_{*}$, the characteristic fractional luminosity $\left(\mathscr{L}^{*}\right)$, namely the characteristic blob contribution of the UV luminosity of their galaxies, increases with redshift, while the number of the characteristic blobs per galaxy $\left(\Phi^{*}\right)$ decreases with redshift. This result shows that the lower the redshift, the fainter (in terms of UV light contribution) the blobs are as well as the larger their numbers are. Although the shift of the FLFs toward the bright end from low to high redshifts could be physical and suggest a transition of the star formation mode with redshift and $M_{*}$ (e.g., as indicated by Mandelker et al. 2014), it is more likely due to an observational effect: the blending of blobs. The faint 
blobs are hard to detect individually at high redshift as well as in low-mass galaxies due to the low spatial resolution in physical length at high redshifts and the small size of the galaxies. If detected blended, they will shift the FLF toward the bright side and suppress the number of the faint blobs, resulting in a decline of the number of the faint blobs toward high redshift and lowmass galaxies as seen in Figure 8.

\section{A PHYSICAL DEFINITION OF CLUMPS}

The issue of the blending of blobs also raises a question: are these small blobs simply blended star-forming regions similar to those seen in nearby disk or spiral galaxies? This reaffirms the problem faced by any clump definition based on the appearance of galaxies: the natures of thus defined clumps change with the redshift and size of galaxies due to observational effects.

In order to understand to what extent our detected blobs can be statistically described by the counterparts of local starforming regions, we shift a grand design spiral galaxy, M101 (NGC5457), to the redshift of each galaxy in our sample and detect blobs from the redshifted images. We use the SDSS $u$-band image for the test. At the distance of M101 (6.4 Mpc), the spatial resolution of the SDSS image (1".4) is equivalent to about $40 \mathrm{pc}$, sufficient to resolve large H II regions. For each galaxy in our sample, we also shrink the physical effective radius of M101 to match the effective radius of the galaxy. We re-bin and smooth the SDSS images to match, in units of kpc, the pixel size and spatial resolution of our HST/ACS blob detection images. We then re-scale the total flux, in units of Analogueto-Digital Unit (ADU), of the re-binned and smoothed M101 image to match the total flux of each of our sample galaxies in the blob detection band. Therefore, the redshifted M101s are matched to the redshift, size, and apparent surface brightness of each galaxy in our sample. We finally add a fake background fluctuation, whose $1 \sigma$ level is equal to that of our HST/ACS detection images, to the re-scaled M101 image. In this paper, we do not follow the rigid steps of redshifting a galaxy, such as determining the morphological K-correction and cosmological dimming (e.g., Barden et al. 2008), because our purpose is not to study how galaxies with the M101 spectral type and luminosity look at higher redshifts. Instead, our purpose is to study how galaxies with the M101 appearance look at higher redshifts. The scaling of the flux of M101 provides a reasonable shortcut for us (see Conselice 2003, for a detailed description of similar simulation tests).

We run our blob finder on the redshifted M101 images and measure the FLFs of the detected blobs in each redshift and $M_{*}$ bin. The results are shown in Figure 9, overplotted with the observed GOODS-S FLFS, both uncorrected for the detection incompleteness. The figure shows clearly that at $z \leqslant 2$, the faint end of the observed FLF in each $M_{*}$ bin can be well explained by that of the redshifted M101s. This indicates that the faint blobs detected by our automated finder are actually not statistically different from the redshifted local $\mathrm{H}$ II regions, once the local galaxies are matched to the size of the high-redshift galaxies. These faint blobs should be excluded from our definition of clumps. The comparison at $z>2$ is not as conclusive as that at $z \leqslant 2$ due to the large Poisson error bars of the observed functions. But still, we see a hint that the redshifted local H II regions can explain a large fraction of the faint end of the observed functions, which suggests that similar observational effects of blurred and blended local $\mathrm{H}$ in regions are also present at $z>2$.
In this paper, we define clumps as blobs whose fractional luminosities are significantly higher than that of redshifted star-forming regions of nearby large spiral galaxies. Particularly, we choose a threshold where the observed FLF is $\sim 3 \sigma$ higher than the FLF of the redshifted M101s. This threshold (where the dashed curves cross the red shaded histograms in Figure 9) changes slightly among different (redshift, $M_{*}$ ) bins. For simplicity, we choose the threshold as $L_{\text {blob }} / L_{\text {galaxy }}=0.08$ and thus define clumps as blobs whose UV luminosity is brighter than $8 \%$ of the total UV luminosity of the galaxies (as shown by the vertical dashed lines in Figure 9). This definition of clumps takes into account the observational effects due to the sensitivity and resolution as well as the change of size of galaxies with redshift and $M_{*}$. Therefore, it defines clumps in a more physical way than the appearance of galaxies and can be easily applied to galaxies at different redshifts regardless of the observational effects.

We also test the $3 \sigma$ threshold of our clump definition by using other nearby spiral galaxies and find that the threshold is only mildly changed. We repeat the above test by redshifting the GALEX NUV (spatial resolution of 5".0) images of M83 and M33. The physical resolution is $\sim 100 \mathrm{pc}$ and $\sim 23 \mathrm{pc}$ at the distance of M83 (4.61 Mpc) and M33 ( $\sim 0.9 \mathrm{Mpc})$. For M83, the $3 \sigma$ thresholds in all (redshift, $M_{*}$ ) bins are quite close to those in the M101 test with a different of at most 0.1 dex, except for in the $z=0.5-1.0$ and $\log \left(M_{*} / M_{\odot}\right)<9.8 \mathrm{bin}$, where the M83 threshold is 0.5 dex smaller than the M101 threshold. For M33, the $3 \sigma$ thresholds are systematically smaller than those of the M101 test by $0.2-0.3$ dex in almost all (redshift, $M_{*}$ ) bins. In our later analyses, we keep using the value of $L_{\text {blob }} / L_{\text {galaxy }}=8 \%$ (the vertical dashed lines in Figure 9) that is derived from the M101 test as the default definition. We will also discuss how $f_{\text {clumpy }}$ changes if we use an aggressive definition of $L_{\text {blob }} / L_{\text {galaxy }}=0.05$ or a conservative definition of $L_{\text {blob }} / L_{\text {galaxy }}=0.1$.

Our clump definition uses the $\mathrm{H}$ II regions of nearby large spiral galaxies as the null hypothesis and rejects it once the event of a blob with $>8 \%$ UV fractional luminosity happens. This definition is appropriate and necessary to exclude "nonclumpy" galaxies, because the purpose of this paper is to carry out a statistical census of clumpy galaxies. It is, however, important to note that using some nearby galaxies as the null hypothesis does not mean all local galaxies are "non-clumpy." In fact, Elmegreen et al. (2009b) carried out similar tests of redshifting local galaxies and found that clumpy galaxies at intermediate to high redshifts resemble local dwarf irregulars in terms of morphology, number of clumps, and relative clump brightness. Therefore, a large fraction of local low-mass galaxies also contain clumps. Our later result (Figure 10) also confirms this point. The purpose of this paper is not to distinguish high-redshift clumps from local clumps. It is to distinguish clumps from non-clumps (i.e., small blobs and small $\mathrm{H}$ II regions). The redshifted local dwarf irregulars cannot serve as a null hypothesis to reject "non-clumpy" galaxies in statistics, although they are excellent high-resolution counterparts to study the physical properties of high-redshift clumpy galaxies (Elmegreen et al. 2009b).

\section{FRACTION OF CLUMPY GALAXIES}

\subsection{Clumpy Fraction}

One of the main results of this paper-the fraction of clumpy galaxies among SFGs $\left(f_{\text {clumpy }}\right)$ in a given $M_{*}$ and redshift bin-is shown in Figure 10. Here clumpy galaxies are defined 


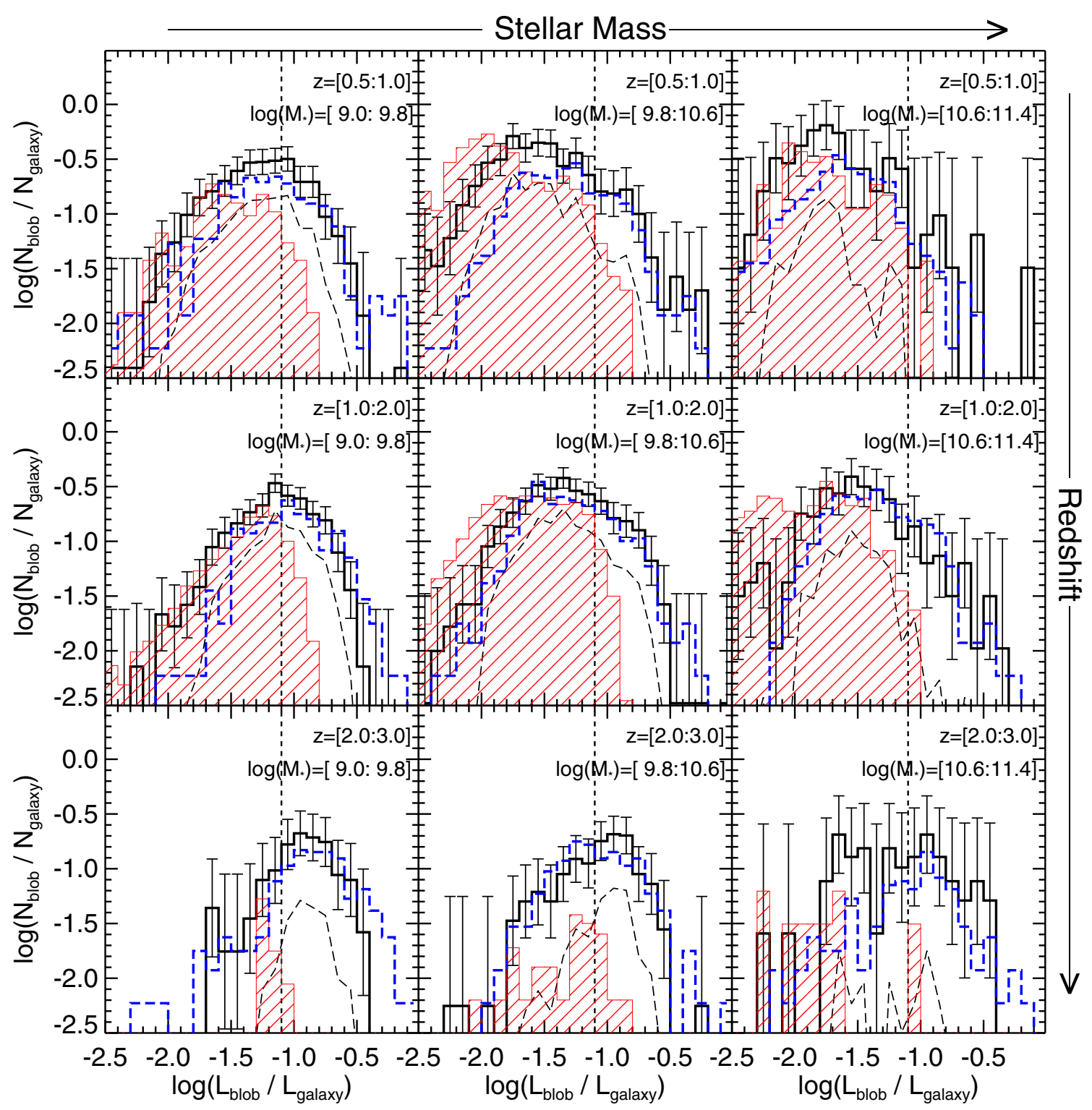

Figure 9. Definition of clumps. In each panel, the fractional luminosity function of GOODS-S (not corrected for the detection incompleteness) is shown by the black histogram with error bars from the Poisson error. The black dashed curve shows the lower $3 \sigma$ level of the error bars. The red shaded region shows the fractional luminosity function of blobs detected from the fake redshifted M101 galaxies. The vertical dashed line shows our definition of clumps: blobs brighter than the line are defined as clumps. The blue dashed histogram shows the fractional luminosity function of blobs detected in the redshifted fiducial galaxies $\left(9.8<\log \left(M_{*} / M_{\odot}\right)<10.6\right.$ and $0.5<z<1.0$, see Section 5 for details).

as galaxies that contain at least one off-center $\left(d_{b}>0.5 r_{e}\right)$ clump as defined in Section 5. We measure the fraction and its uncertainty (Poisson errors from number counts) separately for GOODS-S and UDS. Each color point in the figure is the errorweighted average of the GOODS-S and UDS results. We also show the fractions of the two fields as the hats of the error bar of each data point. Therefore, the error bars in the figure reflect the field variance instead of the statistical uncertainty. The errors of the GOODS-S and UDS fractions are not shown in the figure, but their relative strength can be inferred from the distance of each data point to the two hats of its error bar.

The redshift evolution of $f_{\text {clumpy }}$ changes with $M_{*}$ of the galaxies (the upper left panel of Figure 10). Low-mass galaxies $\left(\log \left(M_{*} / M_{\odot}\right)<9.8\right)$ keep an almost constant $f_{\text {clumpy }}$ around $55 \%$. For intermediate-mass galaxies $\left(9.8<\log \left(M_{*} / M_{\odot}\right)<\right.$ 10.6), $f_{\text {clumpy }}$ remains almost constant around $45 \%$ from $z \sim 3$ to $z \sim 1.5$, and then gradually drops to $\sim 30 \%$ at $z \sim 0.5$. For massive galaxies $\left(10.6<\log \left(M_{*} / M_{\odot}\right)<11.4\right), f_{\text {clumpy }}$ also keeps a constant of $\sim 50 \%$ from $z \sim 3$ to $z \sim 2$, but then quickly drops to $\sim 15 \%$ at $z \sim 0.5$.

We also show $f_{\text {clumpy }}$ under an aggressive $\left(L_{\text {blob }} / L_{\text {galaxy }}=\right.$ $0.05)$ and a conservative clump definition $\left(L_{\text {blob }} / L_{\text {galaxy }}=0.1\right)$ in the upper left panel of Figure 10. The general trend of the $f_{\text {clumpy }}$-redshift relation of each mass range is not significantly affected by the different definitions. The normalization of the relations, however, is scaled up (down) by a factor of 1.2-1.3 for the aggressive (conservative) definition.

The dependence of $f_{\text {clumpy }}$ on $M_{*}$ changes with redshift as well (the top right panel of Figure 10). In general, $f_{\text {clumpy }}$ decreases with $M_{*}$ in all redshift bins, but the slope of the trend depends on the redshift. The lower the redshift, the steeper the slope (i.e., the faster $f_{\text {clumpy }}$ decreases with $M_{*}$ ).

It is important to note that the above trends (top panels of Figure 10) are based on the direct number count of clumps 


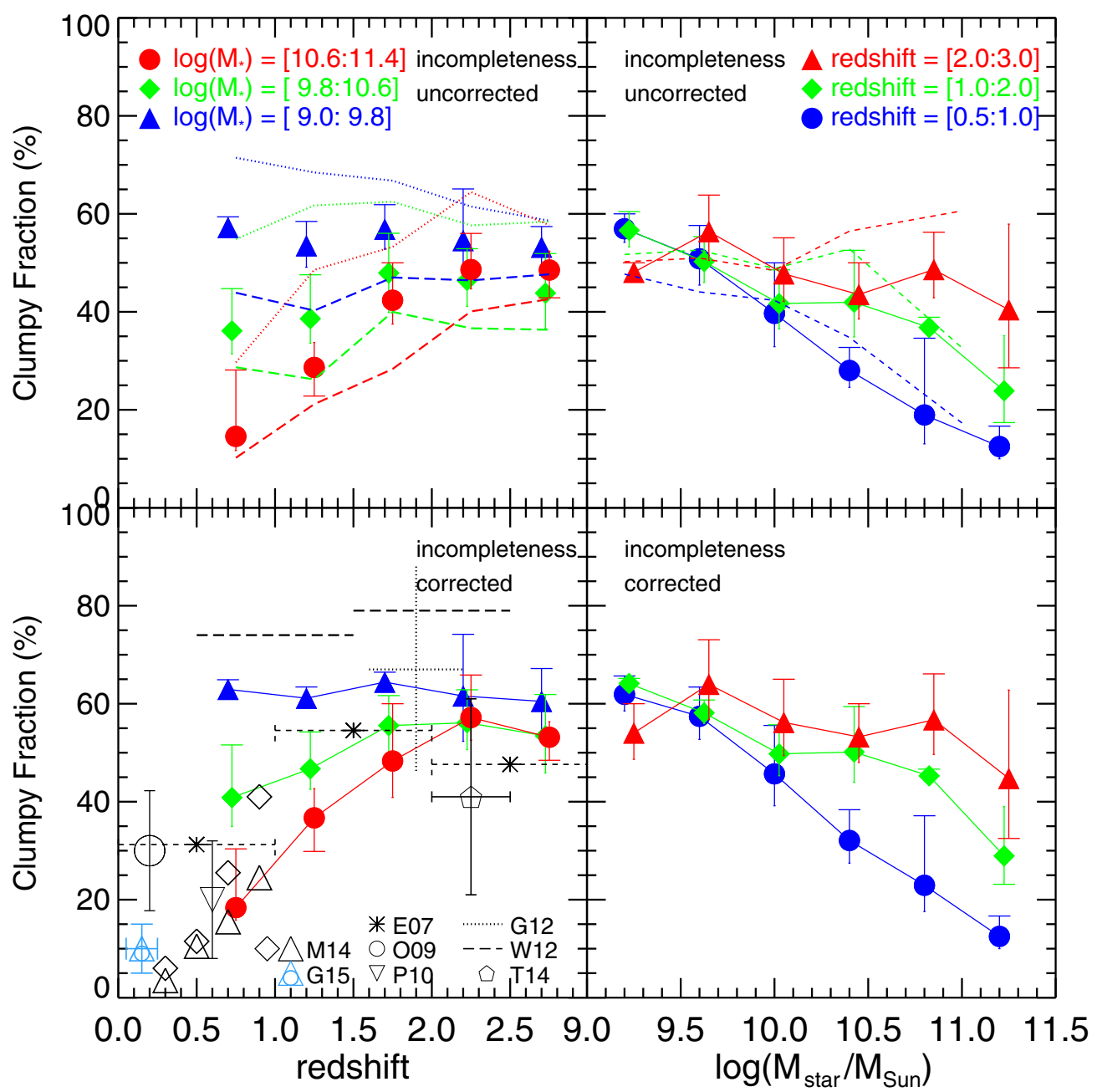

Figure 10. Fraction of star-forming galaxies with at least one off-center UV clump in different redshift and $M_{*}$ bins. The upper panels show the results without correcting for the detection incompleteness, while the lower panels show the results with correcting for the incompleteness through Equation (2). Each colored point is the error-weighted average of the GOODS-S and UDS results. The hats of the upper and lower error bars of each data point have different lengths: the longer hat shows the fraction of GOODS-S, while the shorter one shows that of UDS. The errors of GOODS-S and UDS fractions are not shown, but the relative errors between the two fields can be inferred from the distances of each data point to the two hats of its error bar. In the upper left panel, dashed and dotted lines show $f_{\text {clumpy }}$ under an aggressive $\left(L_{\text {blob }} / L_{\text {galaxy }}=0.05\right)$ and a conservative $\left(L_{\text {blob }} / L_{\text {galaxy }}=0.1\right)$ clump definitions, respectively. The color of each dashed or dotted line matches the color of the symbols to show its $M_{*}$ range. In the upper right panel, dashed lines show $f_{\text {clumpy }}$ measured through comparing real galaxies with redshifted fiducial galaxies to take into account the clump/blob blending effects (see Section 7.1 for details). In the lower left panel, several measurements of $f_{\text {clumpy }}$ from other studies are also plotted. The summary of the previous results is given in Table 1 .

without taking into account the incompleteness of our clump detection. As shown in Figures 6 and 7, although the completeness is relatively high for our clumps (i.e., blobs with high $L_{\text {blob }}\left(L_{\text {galaxy }}\right)$, it is still not unity. Therefore, we may underestimate $f_{\text {clumpy }}$ because of the missing clumps. To correct for the incompleteness, we calculate a new $f_{\text {clumpy }}$ using the following formula, assuming the undetected clumps are randomly distributed in the galaxies in our sample:

$$
\begin{aligned}
f_{\text {clumpy }}^{\text {new }}= & f_{\text {clumpy }}^{\text {old }}+\frac{1}{n_{c}}\left(\frac{1}{X}-1\right)\left(f_{\text {clumpy }}^{\text {old }}\right) \\
& -\frac{1}{n_{c}}\left(\frac{1}{X}-1\right)\left(f_{\text {clumpy }}^{\text {old }}\right)^{2}
\end{aligned}
$$

where $f_{\text {clumpy }}^{\text {old }}$ and $f_{\text {clumpy }}^{\text {new }}$ are the clumpy fractions before and after the incompleteness correction is applied, $X$ the clump detection completeness, and $n_{c}$ the average number of clumps in each clumpy galaxy. The second term on the right hand side takes into account the contribution of undetected clumps, while the third term takes into account the fact that some undetected clumps may be in a galaxy that has already been classified as clumpy, in which case the number of clumpy galaxies should not be increased.

The new clumpy fraction $\left(f_{\text {clumpy }}^{\text {new }}\right)$ depends on how many clumps $\left(n_{c}\right)$ a clumpy galaxy has. In the bottom panels of Figure 10, we plot the results with the assumption of $n_{c}=2$. Compared with the top panels, although the amplitudes of $f_{\text {clumpy }}$ in different redshift and $M_{*}$ bins are scaled up by, on average, a factor of $\sim 1.2$, the trends with redshift and $M_{*}$ are almost unchanged by taking into account the undetected clumps. This is also true if we assume $n_{c}=1$, the most extreme case where each clumpy galaxy only intrinsically has one clump. In that case, the amplitude will be systematically scaled up by a factor $\sim 1.3$, compared to the top panels.

In this paper, we use $f_{\text {clumpy }}$ under our default clump definition $\left(L_{\text {blob }} / L_{\text {galaxy }}=0.08\right)$ and after the incompleteness correction with $n_{c}=2$ as our best measurement (the bottom panels of Figure 10). Overall, low-mass galaxies $\left(\log \left(M_{*} / M_{\odot}\right)<9.8\right)$ keep a constant $f_{\text {clumpy }}$ of $\sim 60 \%$ from $z \sim 3$ to $z \sim 0.5$. Intermediate-mass galaxies $\left(9.8<\log \left(M_{*} / M_{\odot}\right)<10.6\right)$ keep 
Table 1

Summary of Papers and Samples Used for Clumpy Fraction Comparison

\begin{tabular}{|c|c|c|c|c|c|}
\hline Paper & Sample (Number of Galaxies) & Galaxy Mass $\left(M_{\odot}\right)$ & Redshift & Clump Finder & Detection Band \\
\hline E07 (Elmegreen et al. 2007) & Starbursts (1003) & $\mathrm{N} / \mathrm{A}$ & $0<z<5$ & Visual & F775W \\
\hline P10 (Puech 10) & Emission-line galaxies (63) & $>2 \times 10^{10}$ & $\sim 0.6$ & Visual & F435W \\
\hline O09 (Overzier et al. 2009) & Lyman Break Analogs (20) & $10^{9}-10^{10}$ & $\sim 0.2$ & Visual & rest-frame UV \\
\hline G12 (Guo et al. 2012) & Star-forming galaxies (10) & $>10^{10}$ & $1.5<z<2.5$ & Algorithm & F850LP \\
\hline W12 (Wuyts et al. 2012) & Star-forming galaxies (649) & $>10^{10}$ & $0.5<z<2.5$ & Algorithm & rest-frame $2800 \AA$ \\
\hline T14 (Tadaki et al. 2014) & $\mathrm{H} \alpha$-emitting galaxies $(100)$ & $10^{9}-10^{11.5}$ & $2.0<z<2.5$ & Algorithm & F606W \& F160W \\
\hline G15 (Y. Guo et al., in preparation) & Star-forming galaxies (50) & $>10^{10.75}$ & $0.05<z<0.25$ & Algorithm & F225W \\
\hline This work & Star-forming galaxies (3239) & $10^{9}-10^{11.5}$ & $0.5<z<3.0$ & Algorithm & rest-frame $2500 \AA$ \\
\hline
\end{tabular}

an almost constant $f_{\text {clumpy }}$ of $\sim 55 \%$ from $z \sim 3$ to $z \sim 1.5$, and then gradually drops it to $40 \%$ at $z \sim 0.5$. Massive galaxies $\left(10.6<\log \left(M_{*} / M_{\odot}\right)<11.4\right)$ also keep their $f_{\text {clumpy }}$ constant at $\sim 55 \%$ from $z \sim 3$ to $z \sim 2$, but then quickly drop it to $\sim 15 \%$ at $z \sim 0.5$.

\subsection{Comparison with Other Studies}

We compare our $f_{\text {clumpy }}$ with that of other studies in the bottom right panel of Figure 10. The sample, $M_{*}$ range, and clump identification method of each study used in the comparison are summarized in Table 1.

Our $f_{\text {clumpy }}$ of $\log \left(M_{*} / M_{\odot}\right)>9.8$ galaxies shows good agreement with that of Elmegreen et al. (2007, E07) and Puech (2010, P10), both identified clumpy galaxies through visual inspection. E07 didn't specify the $M_{*}$ range of their galaxies, but given their size and surface brightness cuts on the restframe UV images of their galaxies, it is reasonable to compare their results with our $\log \left(M_{*} / M_{\odot}\right)>10$ galaxies. Also, for E07, we only use their categories of clump clusters, spirals, and ellipticals to calculate $f_{\text {clumpy }}$. We exclude chain galaxies, double nuclei, and tadpoles, all of which usually have small axial ratios, to match our requirement on the elongation of galaxies. The agreement with the two measurements reinforces our conclusion that $f_{\text {clumpy }}$ in massive galaxies drops from $\sim 50 \%$ at $z>1.5$ to about $20 \%$ at $z \sim 0.5$.

The results of Murata et al. (2014, M14) also show agreement with our $f_{\text {clumpy }}$. M14 measured $f_{\text {clumpy }}$ for more than 20,000 galaxies at $0.2<z<1.0$ in COSMOS. They identify clumpy galaxies through the peak of the contrast between the 1st, 2nd, and 3rd bright peaks in the $\mathrm{F} 814 \mathrm{~W}$ images of the galaxies. Their result at $10.5<\log \left(M_{*} / M_{\odot}\right)<11.0$ galaxies (open triangles) shows good agreement with ours in the highest $M_{*}$ bin (red circles). Their result at $10<\log \left(M_{*} / M_{\odot}\right)<10.5$ (open diamonds) also matches ours in the intermediate $M_{*}$ bin (green diamonds) at $z \sim 1$. At $z<0.75$, however, their $f_{\text {clumpy }}$ of $10<\log \left(M_{*} / M_{\odot}\right)<10.5$ galaxies quickly drops, while we expect, from the extrapolation of our higher-redshift results, a mild drop. More measurements are needed to confirm the trend of the intermediate-mass galaxies at very low redshift.

Our $f_{\text {clumpy }}$ is also statistically consistent with the results of two other studies, given their large errorbars. Tadaki et al. (2014, T14) measured $f_{\text {clumpy }}$ for $100 \mathrm{H} \alpha$-emitting galaxies at $z=2.19$ and $z=2.53$. They identified clumps from both F606W and F160W images, using the clump finder of Williams et al. (1994) and adjusting the control parameters to match visual inspections. Their sample spans a large $M_{*}$ range from $\log \left(M_{*} / M_{\odot}\right)=9.5$ to $\log \left(M_{*} / M_{\odot}\right)=11.0$. Overall, their $f_{\text {clumpy }}$ of $40 \%$ is lower than ours, if we combine all our $M_{*}$ bins together. Guo et al. $(2012$, G12) used an algorithm similar to ours to identify clumpy galaxies in massive galaxies at $z \sim 2$. Their $f_{\text {clumpy }}$ of $67 \%$ is higher than ours, but their sample contains only 15 galaxies, which is biased toward bright, blue, and large galaxies because they only include spectroscopically observed galaxies. Both T14 and G12, however, have large uncertainties in their $f_{\text {clumpy }}$, making their results still statistically consistent with ours.

$f_{\text {clumpy }}$ of Wuyts et al. (2012, W12) are significantly higher than ours. Instead of detecting individual clumps, W12 detect the pixels with excess surface brightness in multi-band images. Here we use their $f_{\text {clumpy }}$ measured in the rest-frame UV detection. Their threshold of clumpy galaxies is quite low compared to our definition here. They required a total UV (2800 ̊) luminosity contribution of $5 \%$ from all clumps to be a clumpy galaxy, while we ask for at least one clump contributing $8 \%$ of the luminosity. As shown by the top left panel of Figure 10, a lower threshold would include a lot of small star-forming regions, which could explain the high $f_{\text {clumpy }}$ of $\mathrm{W} 12$.

We also include the data of our ongoing HST SNAPSHOT program (HST-GO-13309) in Figure 10 as a boundary condition of the clumpy fraction of massive SFGs at $z \sim 0$. The SNAPSHOT program aims to image a representative sample of 136 SDSS galaxies with $\log \left(M_{*} / M_{\odot}\right)>10.75$ and SSFR $>10^{-0.75}$ at $0.05<z<0.25$ with the HST/WFC3 UVIS F225W filter. The details of the program and data reduction will be presented in a future paper (Y. Guo et al., in preparation, G15). Here, we apply our blob finder to 50 galaxies that have been observed so far and identify clumps from them using the same fractional luminosity threshold $(>8 \%)$. The clumpy fraction (the light blue triangle with a circle embedded at $z \sim 0.15$ in the lower right panel of Figure 10) confirms the rapid decline of $f_{\text {clumpy }}$ in massive galaxies - only less than $10 \%$ of massive SFGs at $z \sim 0.15$ contain off-center UV clumps. Among all other $f_{\text {clumpy }}$ compared in Figure 10, this local sample has the closest sample selection, observational effects, and clump identification to our CANDELS sample. Therefore, it provides the most consistent constraint on the end-point of massive $f_{\text {clumpy }}$ evolution.

\subsection{Implications on Clump Formation}

The fraction of clumpy galaxies and its evolution with redshift have important implications for the formation mechanisms of the clumps. In a widely held view based on theoretical works and numerical simulations, the clumps are formed through gravitational instability in gas-rich turbulent disks (e.g., Noguchi 1999; Immeli et al. 2004a, 2004b; Elmegreen et al. 2008; Dekel et al. 2009b; Ceverino et al. 2010, 2012; Dekel \& Burkert 2014). This scenario is supported by the fact that high-redshift galaxies are gas-rich, with gas-to-baryonic fraction of $20 \%$ to $80 \%$ (e.g., Erb et al. 2006; Genzel et al. 2008; Tacconi et al. 2008, 2010; 


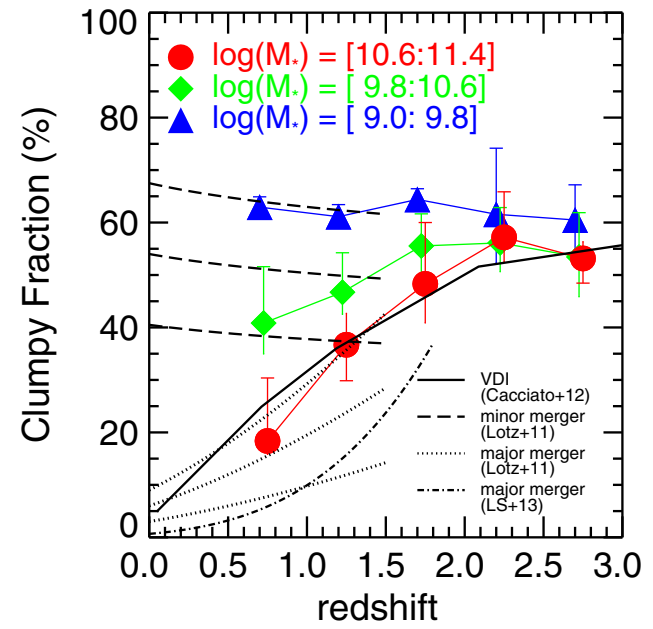

Figure 11. Evolution of the fraction of galaxies with off-center UV clumps as a function of redshift. Color symbols with error bars are identical to those in the lower left panel of Figure 10. The solid black curve shows the of massive disks that are unstable. It is derived by combining the prediction of disk instability in the massive disk of the two-component (gas+star) fiducial model of Cacciato et al. (2012) and the kinematic measurement uncertainty of Kassin et al. (2012). See the text for details. The dashed black lines are the minor merger rate of Lotz et al. (2011), scaled by a merger observability timescale of 1.5, 2, and $2.5 \mathrm{Gyr}$ (from bottom to top). The dotted black lines are the major merger rate of Lotz et al. (2011), scaled by a merger observability timescale of 1, 2, and $3 \mathrm{Gyr}$ (from bottom to top). The dotted-dashed line is the wet major merger fraction of López-Sanjuan et al. (2013).

Förster Schreiber et al. 2009; Daddi et al. 2010), possibly as a result of smooth and continuous accretion of cold gas flow (Kereš et al. 2005; Rauch et al. 2008; Dekel et al. 2009a; Cresci et al. 2010; Steidel et al. 2010; Giavalisco et al. 2011). Genzel et al. (2011) derived the Toomre $Q$ parameter (Toomre 1964) at locations of observed clumps from the $\mathrm{H} \alpha$ velocity map and $\mathrm{H} \alpha$ surface density. They found $Q<1$, meaning gravitationally unstable to collapse, for all clump sites and $Q \sim 1$ throughout the disks, providing evidence for the scenario of the VDI (Dekel et al. 2009b). The kinematic signatures of the clumpy disks, however, can also have an ex-situ origin, such as gas-rich mergers (e.g., Robertson \& Bullock 2008; Puech 2010; Hopkins et al. 2013).

A few possible formation mechanisms of clumps are compared in Figure 11. First, we compare the trend of disk instability predicted by VDI with the evolution of $f_{\text {clumpy }}$ of massive galaxies $\left(\log \left(M_{*} / M_{\odot}\right)>10.6\right)$, testing the idea that clumps are formed in-situ in turbulent disks and are manifestations of gravitational instability in galaxy disks. van der Wel et al. (2014a) found that about $80 \%$ of the massive $\left(10.5<\log \left(M_{*} / M_{\odot}\right)<11.0\right)$ SFG at $0<z<2$ are disky galaxies, which validates the basic assumption of VDI, i.e., the existence of disks. Here, we use the fiducial two-component (gas + stars) model of Cacciato et al. (2012) as representative of VDI. In this model, the massive disk is assumed to be continuously fed by cold gas at the average cosmological rate. The gas forms stars and is partly driven away by stellar feedback. The gravitational energy released by the mass inflow down the gravitational potential gradient drives the disk turbulence that maintains the disk instability. Since the gas is the main driver of instability at all times, once the gas velocity dispersion $\left(\sigma_{\text {gas }}\right)$ is significantly lower than the circular velocity of the disk $\left(V_{\text {circ }}\right)$, the disk can be considered "stable."

Since Cacciato et al. (2012) presents an analytic model with only one realization for each set of parameters, we need to convert their model prediction into a probability (i.e., some fraction of the galaxies) to make a direct comparison with our $f_{\text {clumpy }}$. To this purpose, we assume that the trend of $\sigma_{\text {gas }} / V_{\text {circ }}$ in Cacciato et al. (2012) is the average value for massive SFGs. We then measure the scatter of the $\sigma_{\text {gas }} / V_{\text {circ }}$ from the observations of Kassin et al. (2012), who measured kinematics for a sample of SFG at $z=0.2-1.2$. The $1 \sigma$ scatter of $\sigma_{\text {gas }} / V_{\text {circ }}$ in Kassin et al. (2012) is about 0.5 dex. A Monte Carlo sampling is then carried out based on the average value and scatter to generate a distribution of $\sigma_{\text {gas }} / V_{\text {circ }}$ at different redshifts. We then choose $\sigma_{\text {gas }} / V_{\text {circ }}>1 / 3$ as the threshold of being "unstable disks" (the same threshold of Kassin et al. 2012). We then compare the "unstable" fraction of the Monte Carlo realizations with $f_{\text {clumpy }}$ of our galaxies. The solid line in Figure 11 shows that the "unstable" fraction matches $f_{\text {clumpy }}$ of massive SFGs remarkably well, suggesting that VDI is a likely explanation of the decrease of $f_{\text {clumpy }}$ toward low redshift. It is important to note that, however, the VDI model used here does not directly predict the threshold of forming clumps in an unstable disk. A more direct test of VDI would be either comparing $f_{\text {clumpy }}$ with the clump formation probability predicted by VDI or comparing the characteristic $M_{*}$ of real clumps with that of models (i.e., Toomre Mass predicted by VDI).

The trend of the disk stabilization in Cacciato et al. (2012), however, cannot explain the trend of the intermediate or lowmass galaxies. For the intermediate $\left(9.8<\log \left(M_{*} / M_{\odot}\right)<\right.$ 10.6) galaxies, the slow decrease of the trend at low redshift requires the disk stabilization to be delayed. Although a few variants of the fiducial model of Cacciato et al. (2012) are able to delay the disk stabilization, e.g., by using an extremely high inflow accretion rate or an extremely low star formation efficiency, their trend of disk instability $\left(\sigma_{\text {gas }} / V_{\text {circ }}\right)$ cannot match the trend of the intermediate galaxies at both low and high redshifts simultaneously. Other mechanisms may be needed to explain the redshift evolution of $f_{\text {clumpy }}$ of the intermediate or low-mass galaxies. It is important, however, to note that the models of Cacciato et al. (2012) and similar VDI predictions are made for galaxies with $\log \left(M_{*} / M_{\odot}\right) \sim 11$. Currently, no reliable predictions of VDI on the disk stabilization for lower mass galaxies $\left(\log \left(M_{*} / M_{\odot}\right)<10.5\right)$ are available. The general VDI models (e.g., Bournaud et al. 2011; Dekel \& Burkert 2014), however, do expect less massive galaxies to remain unstable for longer times, because they retain higher gas fractions as a result of the regulation of gas consumption in low-mass galaxies (Dekel \& Silk 1986; Krumholz \& Dekel 2010) and the continuation of gas accretion to low redshift for low-mass halos (Dekel \& Birnboim 2006).

In fact, Elmegreen et al. (2009b) made a qualitative analysis on the validity of the gravitational instability in disks for local dwarf irregulars. They found a striking resemblance of the morphology between high-redshift clumpy galaxies and dwarf irregulars, although the former is intrinsically brighter than the latter by a factor of 10-100. The typical velocity dispersion of local dwarf irregulars is $15 \mathrm{~km} \mathrm{~s}^{-1}$, but their circular velocity is small too, $<100 \mathrm{~km} \mathrm{~s}^{-1}$. As a result, the $\sigma_{\text {gas }} / V_{\text {circ }}$ of dwarf irregulars is actually comparable to that of high-redshift disk galaxies (Förster Schreiber et al. 2009), indicating that they may be subject to the same physical mechanism (VDI) to form disk. Future quantitative VDI models for low-redshift lowmass galaxies would provide more detailed tests to the clump formation in this regime.

Minor mergers, on the other hand, provide a viable explanation of $f_{\text {clumpy }}$ of the intermediate $\left(9.8<\log M_{*} / M_{\odot}<10.6\right)$ 
galaxies at $z<1.5$. Lotz et al. (2011) measured the minor merger rate of $\log \left(M_{*} / M_{\odot}\right)>10$ galaxies at $z<1.5$ : $0.27 \times(1+z)^{-0.1} \mathrm{Gyr}^{-1}$. In order to compare the merger rate with $f_{\text {clumpy }}$, we multiply the merger rate by an observability timescale of 1.5, 2, and 2.5 Gyr (dashed black lines from bottom to top in Figure 11). The comparison shows that if the observability timescale of minor mergers is between 1.5 and 2 Gyr, the minor merger fraction qualitatively matches $f_{\text {clumpy }}$ of $9.8<\log \left(M_{*} / M_{\odot}\right)<10.6$ galaxies. In fact, Lotz et al. (2010) studied the effect of gas fraction on the morphology and timescales of disk galaxy mergers and found that when the gas fraction is higher than $50 \%$, the timescales for morphological disturbances measured by Gini and/or Asymmetry can be as long as or more than $1.5 \mathrm{Gyr}$ for a 9:1 baryonic mass ratio merger. Given the high gas-fraction of galaxies around $\log \left(M_{*} / M_{\odot}\right)=10$ at $z \sim 1$ (e.g., Tacconi et al. 2013), the long observability timescale of minor mergers is feasible. Therefore, minor mergers are a viable explanation of $f_{\text {clumpy }}$ of the intermediate $\left(9.8<\log \left(M_{*} / M_{\odot}\right)<10.6\right)$ galaxies at $z<1.5$. At $z>1.5$ as well as for low-mass $\left(\log \left(M_{*} / M_{\odot}\right)<9.6\right)$ galaxies at all redshifts, since the minor merger rate has not been studied thoroughly (e.g., Newman et al. 2012), it is hard to evaluate the role of minor mergers on clump formation at $z>1.5$.

The connection between minor mergers and clumps can happen in two ways. First, minor mergers are the clumps themselves. Such clumps are called ex-situ clumps, while those formed in the disk are called in-situ clumps. Mandelker et al. (2014) analyzed the cosmological hydro-simulations of Ceverino et al. (2010) and found that about $15 \%$ of clumps are ex-situ clumps (i.e., they have their own dark matter components before merging into the primary galaxies). They also found that ex-situ clumps have higher mass, lower gas fraction, lower specific SFR, and older stellar population than in-situ clumps have. We will carry out a detailed analysis on the properties of clumps in a future paper to test the fraction of ex-situ clumps in observations. Second, minor mergers can induce clump formation in the disks. Minor merger would disturb the cold gas distribution in the disks and increase the gas surface density locally, which then results in a local mini-starburst to form a clump (see the resemblance of clumps and starbursts in Bournaud et al. 2014). Unlike gas-rich major mergers, which induce galaxy-wide star-bursts, each minor merger may only be able to induce local starbursts in a few locations. S. Inoue et al. (in preparation) found that in simulations, clumps can be formed in some stable (Toomre $Q>1$ ) disks. In this case, external stimulation (e.g., minor merger) may be needed to enhance the gravitational instabilities to form clumps.

Another possible clump formation mechanism-major mergers-is unlikely able to explain $f_{\text {clumpy }}$ evolution in any mass range, unless its observability timescale is $\gtrsim 3$ Gyr. Figure 11 shows that only with such a long timescale, is the major merger rate from Lotz et al. (2011) $\left(0.03 \times(1+z)^{1.7} \mathrm{Gyr}^{-1}\right)$ able to match $f_{\text {clumpy }}$ of $\log \left(M_{*} / M_{\odot}\right)>10.6$ galaxies at $z<1.5$. Lotz et al. (2010), however, shows that the timescales for morphological disturbances measured by various merger indicators are all less than 2 Gyr even when the gas fraction of the equal-mass merger is as high as $60 \%$. Longer timescales require even higher gas fractions. At $z \sim 1$, however, Tacconi et al. (2013) show that the gas fraction of massive galaxies is $\lesssim 30 \%$, which implies a much shorter $(\sim 0.5 \mathrm{Gyr})$ observability timescale for massive galaxies. Another measurement of the wet major merger fraction from López-Sanjuan et al. (2013) is also lower than $f_{\text {clumpy }}$ at $0<z<1.8$. Therefore, we conclude that major mergers are unlikely to be a viable explanation for the observed trend of $f_{\text {clumpy }}$.

In summary, VDI predicts a strongly decreasing trend of disk instability toward the present day, which qualitatively matches the evolution of $f_{\text {clumpy }}$ of massive galaxies. The normalization and the slopes of the minor and major merger fractions are uncertain, depending on their observability timescale, especially for the low mass galaxy bin. The comparisons show some level of correspondence with the expectations of a minor merger origin of clumps for intermediate-mass galaxies at $z<1.5$, but appear to strongly disfavor a major merger origin of clumps (observability timescales would need to exceed $3 \mathrm{Gyr}$ ) for all galaxies at $z<1.5$. The effects of both minor and major mergers on the clump formation at $z>1.5$ are still unclear due to the lack of a robust estimate of the merger rates. The expectations of all scenarios would benefit from further more realistic modeling of larger cosmologically motivated samples of galaxies.

\section{CLUMP CONTRIBUTION TO THE REST-FRAME UV LIGHT OF GALAXIES}

\subsection{Clump Contribution to the Rest-frame UV Light of Galaxies}

The clump contribution to the rest-frame UV light per galaxy $\left(C_{\mathrm{UV}}\right)$ can be derived through integrating the FLF: $C_{\mathrm{UV}}=\int n(\mathscr{L}) \mathscr{L} d \mathscr{L}$, where $\mathscr{L}=L_{\text {clump }} / L_{\text {galaxy }}$ and the upper and lower limits of the integration are unity and the threshold of our clump definition (Section 5), respectively. Because our incompleteness-corrected FLFs take into account both "clumpy" and "non-clumpy" galaxies (see Section 4 for the discussion of why we cannot separate them), the $C_{\mathrm{UV}}$ we discuss later is the contribution to the entire population of SFGs averaged over both "clumpy" and "non-clumpy" galaxies.

One issue has to be considered when we measure $C_{\mathrm{UV}}$ : the blending of clumps and blobs in high-redshift and/or lowmass bins, where galaxies tend to have smaller sizes (Figure 2 ). The blob blending gradually shifts the FLF to the bright end, but meanwhile lowers the peak of the FLF. The net result of the two actions is that the number of the clumps are kept more-or-less the same. The clump luminosity is, however, artificially increased due to the blending, which would result in an overestimate of $C_{\mathrm{UV}}$ if we simply integrate the observed FLF. ${ }^{13}$

To solve this issue, we use a different way, instead of integration of the incompleteness-corrected FLF, to calculate $C_{\text {UV }}$. We choose the galaxies with $0.5<z<1.0$ and $9.8<\log \left(M_{*} / M_{\odot}\right)<10.6$ as fiducial templates, assuming (1) the clump-clump or clump-blob blending issue is the least significant in this $\left(M_{*}, z\right)$ bin and (2) galaxies and clumps in this $\left(M_{*}, z\right)$ bin are representative for all galaxies and clumps in our sample. We then rescale the size and apparent magnitude of the template galaxies to match the median size and magnitude (in units of ADU) of galaxies in other $\left(M_{*}, z\right)$ bins. We then run our blob finder on the rescaled galaxies and derive the FLF in each bin. The newly derived FLFs, as shown by blue histograms in Figure 9, tell us the clump contribution if we move the fiducial

\footnotetext{
13 This issue has little effect on the measurement of $f_{\text {clumpy }}$. We confirm this in the top right panel of Figure 10 . In this panel, we measure a new $f_{\text {clumpy }}$ by replacing $C_{\mathrm{UV}}$ in Equations (4) and (3) by $f_{\text {clumpy }}$. The new results (dashed lines) show that the normalization and trend of galaxies at $0.5<z<2$ are almost preserved. Only for massive $\left(\log \left(M_{*} / M_{\odot}\right)>10.6\right)$ galaxies at $2<z<3$, the new $f_{\text {clumpy }}$ apparently deviates from the old $f_{\text {clumpy }}$, suggesting an almost flat $f_{\text {clumpy }}-M_{*}$ relation at $z>2$ and an earlier decline of $f_{\text {clumpy }}$ for massive galaxies starting from $z \sim 3$ in the $f_{\text {clumpy }}-z$ trend.
} 


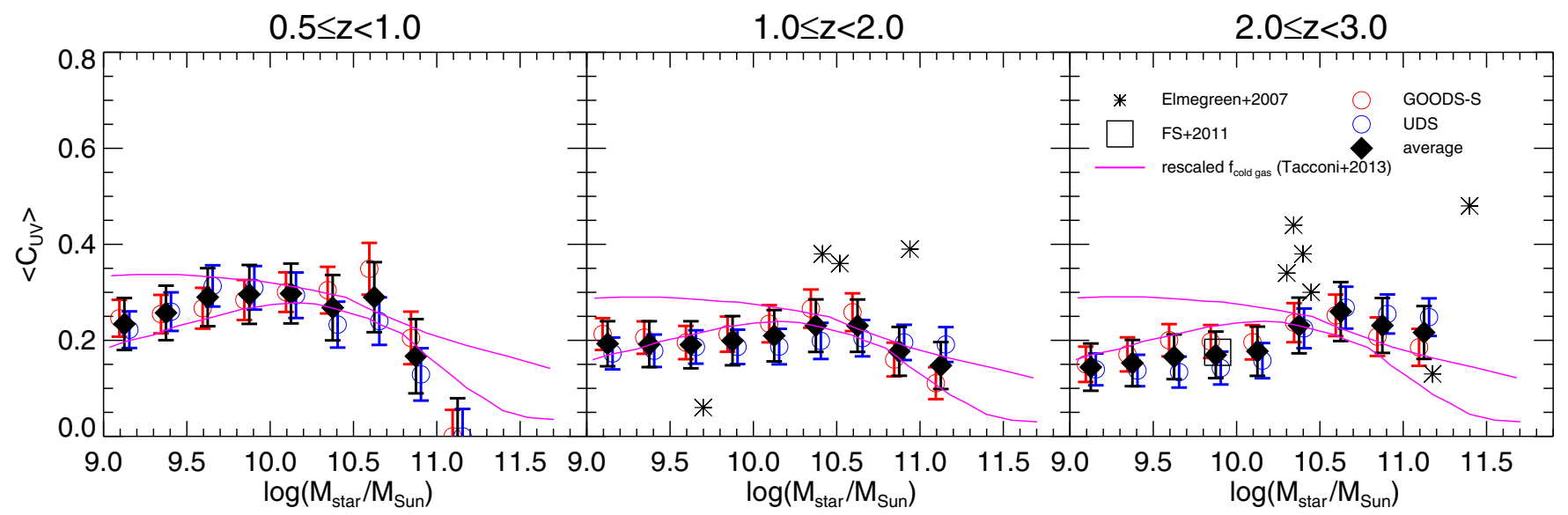

Figure 12. Average clump contribution to the rest-frame UV light $\left(C_{\mathrm{UV}}\right.$, see Section 7.1 for details) of a galaxy as a function of the $M_{*}$ of the galaxy. The contribution is corrected for the detection incompleteness and averaged over all SFGs with or without detected clumps. The results of GOODS-S (red circles and error bars), UDS (blue circles and error bars), and the error-weighted average of the two fields (filled black diamond) are plotted. Measurements from other studies are also shown, as the labels indicate in the third panel. The ranges of the molecular gas fraction of Tacconi et al. (2013) are normalized to match our fraction at $\log \left(M_{*} / M_{\odot}\right)=10.5$ and overplotted with violet curves.

galaxies to other $\left(M_{*}, z\right)$ bins, taking into account both the size change as well as the cosmological dimming.

For each $\left(M_{*}, z\right)$ bin, its actual clump contribution, $C_{\mathrm{UV}}\left(M_{*}, z\right)$, is then derived by scaling the clump contribution of the fiducial bin, $C_{\mathrm{UV}}\left(M_{* F}, z_{F}\right)$, by a ratio $\Upsilon$ :

$$
C_{\mathrm{UV}}\left(M_{*}, z\right)=\Upsilon C_{\mathrm{UV}}\left(M_{* F}, z_{F}\right) .
$$

$\Upsilon$ is defined as

$$
\begin{aligned}
\Upsilon & \equiv \frac{c_{\mathrm{UV}}^{\prime}\left(M_{*}, z\right)}{c_{\mathrm{UV}}^{\prime, r s}\left(M_{* F}, z_{F}\right)}=\frac{X c_{\mathrm{UV}}^{\prime}\left(M_{*}, z\right)}{X c_{\mathrm{UV}}^{\prime, r s}\left(M_{* F}, z_{F}\right)} \\
& =\frac{C_{\mathrm{UV}}\left(M_{*}, z\right)}{C_{\mathrm{UV}}^{r s}\left(M_{* F}, z_{F}\right)},
\end{aligned}
$$

where $c_{\mathrm{UV}}^{\prime}\left(M_{*}, z\right)$ and $c_{\mathrm{UV}}^{\prime, r s}\left(M_{* F}, z_{F}\right)$ are the clump contributions of the FLF in the $\left(M_{*}, z\right)$ bin and of the redshifted fiducial FLF before the detection incompleteness is corrected. We use the ratio of $c_{\mathrm{UV}}^{\prime}\left(M_{*}, z\right)$ and $c_{\mathrm{UV}}^{\prime, r s}\left(M_{* F}, z_{F}\right)$ to measure $\Upsilon$. The incompleteness correction factor in the $\left(M_{*}, z\right)$ bin $(X$ in the above equation) is the same for both the actual and the redshifted fiducial FLFs, because both have the same matched observational effects and galaxy surface brightness distributions. Therefore, the measured $\Upsilon$ is equal to the ratio of $C_{\mathrm{UV}}\left(M_{*}, z\right)$ and $C_{\mathrm{UV}}^{r s}\left(M_{* F}, z_{F}\right)$, i.e., the ratio of the clump contributions of the FLF in the $\left(M_{*}, z\right)$ bin and of the redshifted fiducial FLF $a f$ ter the detection incompleteness is corrected. If $\Upsilon$ is larger than one, the clump contribution in the $\left(M_{*}, z\right)$ bin is higher than that in the fiducial bin, and vice versa. Since the intrinsic clump contribution in the fiducial bin would not change when the galaxies are redshifted, we have $C_{\mathrm{UV}}\left(M_{* F}, z_{F}\right)=C_{\mathrm{UV}}^{r s}\left(M_{* F}, z_{F}\right)$, which leads Equation (4) back to Equation (3).

It is important to note that Figure 9 is only for illustrating the comparison between actual and redshifted FLFs. In reality, we use finer $\left(M_{*}, z\right)$ bins to derive $C_{\mathrm{UV}}\left(M_{*}, z\right)$. Also, we do not apply this method to galaxies with $\log \left(M_{*} / M_{\odot}\right)>10.6$ at $z<1$, because their sizes are larger than that of the fiducial galaxies. The blending issue is even less a problem for them than for the fiducial galaxies.

An example of how this method overcomes the clump-clump or clump-blob blending issue can be seen from the $2.0<$ $z<3.0$ and $9.0<\log \left(M_{*} / M_{\odot}\right)<9.8$ bin in Figure 9. If we simply integrate the incompleteness-corrected FLFs (black histograms) of both this bin and the fiducial bin down to the clump definitions, the clump contribution of this bin would be larger than that of the fiducial bin. The blue histogram in the figure, however, shows that once we redshifted the fiducial galaxies to match the size and magnitude of the galaxies in this $\left(M_{*}, z\right)$ bin, their FLF is actually significantly higher than that of the real galaxies in this bin in the bright end, implying that the clump contribution of real galaxies in this bin is actually lower than that in the fiducial bin. The reason for the apparently higher clump contribution of this high-redshift bin is actually due to the clump-clump or clump-blob blending, which makes clumps look large/bright.

Figure 12 shows $C_{\mathrm{UV}}$ as a function of $M_{*}$ in three redshift bins. $C_{\mathrm{UV}}$ does not change monotonically with $M_{*}$. It increases with the $M_{*}$ from $\log \left(M_{*} / M_{\odot}\right) \sim 9.0$ to $\log \left(M_{*} / M_{\odot}\right) \sim 10.0$, and then reaches a broad peak around $\log \left(M_{*} / M_{\odot}\right) \sim 10.5$, and quickly drops at $\log \left(M_{*} / M_{\odot}\right)>10.8$.

We compare our measurements with other studies in Figure 12. Elmegreen \& Elmegreen (2005) measured the light contribution of ten galaxies at $1<z<3$ in the $i$-band $(\mathrm{F} 775 \mathrm{~W})$ in the HUDF. Förster Schreiber et al. (2011) also measured the F775W light contribution of one galaxy in their $z \gtrsim 2$ sample. Both studies counted only "clumpy" galaxies. It is then not surprising that their clump contributions are higher than ours. At $1<z<3$, the values of Elmegreen \& Elmegreen (2005) are indeed systematically higher than the average of our two fields. The median of their work is about two times higher than our value in the $\log \left(M_{*} / M_{\odot}\right) \sim 10.5$ mass bin. The value of the single galaxy of FS11, in the $\log \left(M_{*} / M_{\odot}\right) \sim 9.9$ mass bin, is slightly higher than our measurements in both fields. If we assume that all our clump contribution is from the detected "clumpy" galaxies and use $f_{\text {clumpy }}=0.5$ from Figure 11, our clump contribution at $10<\log \left(M_{*} / M_{\odot}\right)<11$ and $1<z<3$ should be scaled by a factor of two, when we only consider the clump contributions to clumpy galaxies. Our $C_{\mathrm{UV}}$ is then consistent with that of the two studies. Overall, our measurements are broadly consistent with those of other studies, but the small samples of other studies prevent us from making robust comparison at all redshift and $M_{*}$ ranges. 


\subsection{A Possible Link to Molecular Gas Fraction}

The trend of $C_{\mathrm{UV}}$ as a function of the galaxy $M_{*}$ could be mostly driven by the molecular gas fraction of the galaxies. The physical link could be established in two ways. First, UV bright clumps are shown to be regions with enhanced specific star-formation rates (Förster Schreiber et al. 2011; Guo et al. 2012; Wuyts et al. 2012, 2013). Since the star formation activity is controlled by the amount of molecular gas in galaxies, it is natural to assume that $C_{\mathrm{UV}}$, a signal of the strength of star formation in galaxies, reflects the molecular gas fraction in these galaxies. Second, an important condition of forming clumps is gas-rich galaxies in both the VDI and merger scenarios. Therefore, how important the clumps are in the galaxies, namely the fraction of the stars that formed in the giant clumps, is determined by the cold gas fraction of the galaxies. ${ }^{14}$

To test the possible link between the gas fraction and $C_{\mathrm{UV}}$, we overplot the molecular gas fraction of Tacconi et al. (2013) in Figure 12. Tacconi et al. (2013) measured the molecular gas fraction for 50 SFGs at $z \sim 1-1.5$. Here we use their result that takes into account the incompleteness of the PHIBSS survey. The gas fraction of galaxies below their detection limit was derived by using an empirical gas depletion timescale. Tacconi et al. (2013) also showed that once the gas fraction is normalized to the value at $M_{*}=10^{10.5} M_{\odot}$, the gas fraction $-M_{*}$ trend of $z=$ 0 SFGs and that of $z \sim 1-1.5$ SFGs show remarkable agreement with the redshift dependence almost fully removed. Therefore, assuming that the gas fraction is responsible for the clump contribution $C_{\mathrm{UV}}\left(M_{*}, z\right)$, we simply rescale the gas fraction at $z=1$ in order to match $C_{\mathrm{UV}}$ in all redshift bins:

$$
\begin{aligned}
C_{\mathrm{UV}}\left(M_{*}, z\right) & =A \times f_{\mathrm{gas}}\left(M_{*}, z\right) \\
& =a(z) \times \frac{f_{\mathrm{gas}}\left(M_{*}, z=1\right)}{f_{\mathrm{gas}}\left(10^{10.5} M_{\odot}, z=1\right)},
\end{aligned}
$$

where $a(z)$, the normalization factor, is redshift dependent and may be determined by other physical mechanisms.

The normalized molecular gas fraction shows agreement with our $C_{\mathrm{UV}}$ in the intermediate and massive $M_{*}$ bins, both showing a quick drop above $\log \left(M_{*} / M_{\odot}\right)=10.6$. This supports our speculation that $C_{\mathrm{UV}}$ is mostly driven by the molecular gas fraction of galaxies, at least for intermediate and massive galaxies at $0.5<z<2$. Since There are almost no robust measurements of the gas fraction $-M_{*}$ relation at $2<z<3$, it is unclear if our comparison with the normalized lower-redshift molecular gas fraction is still valid at $2<z<3$.

The link between $C_{\mathrm{UV}}$ and the molecular gas fraction for massive galaxies at $0.5<z<2$ is also consistent with our previous result that VDI is likely responsible for the quick drop of $f_{\text {clumpy }}$ of massive galaxies toward low redshift. VDI predicts that the lower the cold gas surface density, the more stable the disk. For massive galaxies with a given $M_{*}$ (e.g., $\left.10^{11} M_{\odot}\right)$, toward low redshift, the cold gas fraction decreases (Saintonge et al. 2011) but the size of galaxies slightly increases (van der Wel et al. 2014b). Therefore, the cold gas surface density decreases and hence results in a stable disk where clumps are hard to form. In fact, the model of Cacciato et al. (2012) used in Figure 11 takes into account of the gas surface density decreases. In their model, the Toomre $Q$ parameter at

\footnotetext{
14 Here we use molecular gas fraction to represent the cold gas fraction. This is valid if the molecular gas mass dominates the cold gas mass, which is true for galaxies at $z>1.5$ (Obreschkow \& Rawlings 2009) as well as for massive galaxies (>a few times $10^{10} M_{\odot}$ ) at $z \sim 1$ (Tacconi et al. 2013).
}

low redshift is dominated by the stellar component rather than by the gas component.

It is difficult to draw firm conclusion on whether the same $C_{\mathrm{UV}}-$ molecular gas fraction link is still valid for low-mass $\left(<10^{10} M_{\odot}\right)$ galaxies due to a few reasons. First, when measuring $C_{\mathrm{UV}}$, we assume that (1) clumps in galaxies at different $\left(M_{*}, z\right)$ bins are self-similar and (2) the clump FLF in the fiducial bin is representative for the intrinsic FLFS of all other bins. The two assumptions may break down preferentially for low-mass galaxies because of their small sizes (Figure 2). Second, there is no robust measurement of the molecular gas fraction in low-mass galaxies. Tacconi et al. (2013) only measured molecular gas fraction for massive galaxies. Their fraction for galaxies with $\log \left(M_{*} / M_{\odot}\right)<10$ is calculated through $f_{\text {gas }}=1 /\left(1+1 /\left(\operatorname{SSFR} \times t_{\text {depl }}\right)\right)$, where SSFR is the average specific SFR of the star-forming main sequence in literature and $t_{\mathrm{depl}}$ is the gas depletion timescale. Saintonge et al. (2011) found that $t_{\text {depl }}$ is positively correlated with $M_{*}$, but Tacconi et al. (2013) set a constant $t_{\text {depl }}=0.7 \mathrm{Gyr}$ for all galaxy masses. Therefore, lowmass galaxies should have lower $t_{\mathrm{depl}}$ and hence lower inferred gas fraction than in Tacconi et al. (2013). A further measurement of molecular gas fraction for low-mass galaxies is needed to investigate the $C_{\mathrm{UV}}-$ molecular gas fraction in the low-mass end, which can be used to study the physical mechanisms of regulating star formation (e.g., radiation pressure feedback discussed by Moody et al. 2014).

\subsection{Clump Contribution to the Cosmic Star Formation}

We derive the clump contribution to the SFR of SFGs $\left(C_{\mathrm{SFR}}\right)$ from $C_{\mathrm{UV}}$. The rest-frame UV light is a good tracer of SFR if the dust extinction is well understood. In our case, we need to know the dust extinction of both clumps and intra-clump regions. Such dust extinction maps can be measured through spatially resolved multi-band SEDs (e.g., Guo et al. 2012; Wuyts et al. 2012, 2013). We leave accurate measurements of clump properties to a future paper. Instead, we try to use a simplified way to derive a first order estimate of $C_{\mathrm{SFR}}$.

We assume that the dust extinction difference between clumps and galaxies is $\Delta A_{V}=A_{V}$ (galaxy) $-A_{V}$ (clump) $=$ 0.6 and scale down $C_{\mathrm{UV}}$ by a factor of $f_{\text {dust }}=$ $10.0^{-0.4 \times \Delta A_{V} / R_{V} \times k(2500 \AA)}=0.34$, where $R_{V}$ and $k(2500 \AA)$ are derived from the Calzetti Law (Calzetti et al. 2000). This prescription is predicated on two assumptions. First, since we only use galaxies with axial ratio $q>0.5$, we assume the dust extinction in our sample is quite small, with a median value of $A_{V}=0.6$. Second, as shown by Wuyts et al. (2013), UV bright clumps are regions with smaller dust extinction than the intraclump regions. Based on our previous study (Guo et al. 2012), we believe that the extinction difference of $\Delta A_{V}=0.6$ is valid for most of the clumps and galaxies. On the other hand, if we assume that the dust extinction of the clumps and the overall galaxies are the same, i.e., $\Delta A_{V}=A_{V}($ galaxy $)-A_{V}($ clump $)=0.0$, $C_{\mathrm{SFR}}$ would be equal to $C_{\mathrm{UV}}$, namely, $f_{\text {dust }}=1$. In this case, all values of $C_{\mathrm{SFR}}$ in our later discussions should be scaled up by a factor of $\sim 3$.

The redshift evolution of $C_{\mathrm{SFR}}$ for different mass bins is shown in Figure 13. For low- and intermediate-mass galaxies $\left(9<\log \left(M_{*} / M_{\odot}\right)<10.6\right), C_{\mathrm{SFR}}$ increases from $6 \%$ at $z \sim 3$ to $10 \%$ at $z \sim 0.5$. For massive galaxies $\left(\log \left(M_{*} / M_{\odot}\right)>10.8\right)$, $C_{\text {SFR }}$ shows a mild decrease from $8 \%$ at $z \sim 3$ to $5 \%$ at $z \sim 0.5$.

It is interesting to compare our results with other studies. Both Guo et al. (2012) and Elmegreen \& Elmegreen (2005) studied clumps in massive galaxies $\left(\log \left(M_{*} / M_{\odot}\right)>10\right)$ at $z>1.5$. 


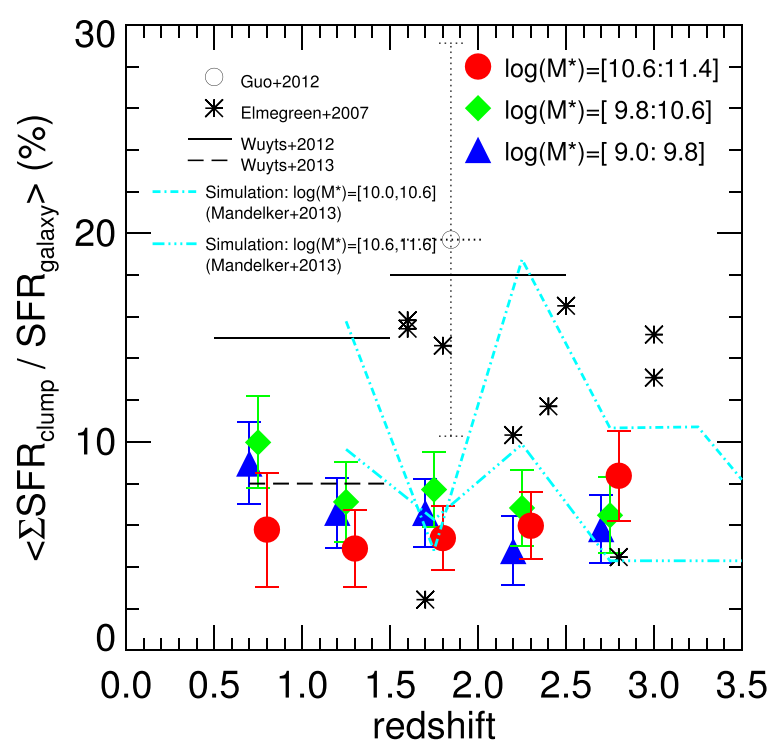

Figure 13. Clump contribution to the SFR of galaxies. The colored points with error bars are from our results, divided into different galaxy $M_{*}$ bins, as the labels show. Measurements from other studies are also overplotted. Note that our study and Wuyts et al. $(2012,2013)$ measured the clump contribution to the SFR of all galaxies, both clumpy and non-clumpy, while Guo et al. (2012) and Elmegreen \& Elmegreen (2005) measured the clump contribution to the SFR of only clumpy galaxies. The results of Mandelker et al. (2014), who analyzed the cosmological hydro-simulation of Ceverino et al. (2010), are also plotted, after being converted to the contribution to all galaxies.

Since their samples only contain clumpy galaxies, their $C_{\mathrm{SFR}}$ should be higher than our value. Guo et al. (2012) measured the SFR of each clump through spatially resolved SED-fitting. We include the contribution of all off-center clumps from their sample. Their $C_{\text {SFR }}$ (circle in Figure 8) is about $20 \%$, a factor of 2.5 higher than our values of massive $\left(\log \left(M_{*} / M_{\odot}\right)>10\right)$ galaxies at $z \sim 2$. Elmegreen \& Elmegreen (2005) only measured the F775W light fraction from clumps. We adopt the above assumption $\Delta A_{V}=A_{V}$ (galaxy) $-A_{V}$ (clump) $\sim A_{V}$ to convert their UV light fraction into SFR fraction. Their values (stars in the figure) are about two times higher than ours. The higher $C_{\mathrm{SFR}}$ of both papers is broadly consistent with our expectation, because they only consider the contribution to "clumpy" galaxies, while we consider the contribution to all SFGs.

Wuyts et al. (2012) measured $C_{\mathrm{SFR}}$ of $\log \left(M_{*} / M_{\odot}\right)>10$ galaxies at $0.5<z<2.5$ in the GOODS-S field through spatially resolved SED-fittings. Because their sample contains both clumpy and non-clumpy galaxies, their $C_{\mathrm{SFR}}$ provides a direct comparison to our study. Here we only quote their measurements based on the rest-frame $2800 \AA$ clump detection. Their values are higher than ours by a factor of $\sim 1.5$. It is important to note that Wuyts et al. (2012) did not detect each individual clump. Instead, they focused on regions with excess surface brightness and only statistically separated clump pixels from disk and bulge pixels. Also, they did not subtract background when measuring clump fluxes. Both could contribute to the discrepancy. Wuyts et al. (2013) revisited the clump contribution to SFR by combining CANDELS and 3D-HST (Brammer et al. 2012). They derived the SFR of clump and disk pixels from dust-corrected $\mathrm{H} \alpha$ luminosity. At $0.7<z<1.5$, their $C_{\mathrm{SFR}}$ of $\log \left(M_{*} / M_{\odot}\right)>10$ galaxies drops from $15 \%$ in Wuyts et al. (2012) to $9 \%$, and is now in very good agreement with ours.

\subsection{Comparison with Cosmological Hydrodynamic Simulations}

We compare our $C_{\mathrm{SFR}}$ with that of the state-of-art numerical simulations. Mandelker et al. (2014) analyzed a large sample of simulations, generated by the same code of Ceverino et al. (2010), to detect clumps from snapshots of 3D gas density. In Figure 13, we overplot (in cyan) their $C_{\mathrm{SFR}}$ for two $M_{*}$ bins. The curves are made by scaling down $C_{\mathrm{SFR}}$ of "clumpy" galaxies in Mandelker et al. (2014) by $f_{\text {clumpy }}$ in their simulations. Their $C_{\mathrm{SFR}}$ shows no clear trend from $z=3.5$ to $z=1.0$. The large fluctuations of the curves, which reflect the uncertainty levels of their measurements, prevent us from drawing firm conclusions. Overall, their $C_{\mathrm{SFR}}$ of intermediatemass $\left(10.0<\log \left(M_{*} / M_{\odot}\right)<10.6\right)$ galaxies seems higher than our measurements, while their $C_{\mathrm{SFR}}$ of massive galaxies $\left(10.6<\log \left(M_{*} / M_{\odot}\right)<11.6\right)$ agrees with ours within the uncertainties.

But the definitions of clumps in our paper and Mandelker et al. (2014) are different. Besides identifying clumps from 3D gas snapshots, Mandelker et al. (2014) included lots of small clumps with SFR contribution less than a few percent of that of the galaxies. The different clump definitions could make the above comparison unfair. A proper way to compare observations with simulations is to generate simulated images that match all the observational effects of the real images. An example can be found in Moody et al. (2014). The same set of simulations has been run through SUNRISE (Jonsson 2006; Jonsson et al. 2010; Jonsson \& Primack 2010) to calculate the radiative transfer and then generate light images in given observational bands. These light images are then downgraded to match the resolution and noise level of real CANDELS images (called "CANDELization," see Snyder et al. 2014 and M. Mozena et al., in preparation). In a separate paper, we will run our clump finder on the "CANDELized" simulation images to make a direct comparison of clumpy galaxies between observations and simulations.

\section{CONCLUSIONS AND DISCUSSIONS}

In this paper, we measure the fraction of clumpy galaxies in SFGs and the clump contributions to the rest-frame UV light and SFR of SFGs in the CANDELS/GOODS-S and UDS fields. Our mass-complete sample consists of 3239 SFGs (SSFR > $0.1 \mathrm{Gyr}^{-1}$ ) at $0.5<z<3$ with axial ratio $q>0.5$. We propose a definition of the UV-bright clumps in a way that is more physical than the appearance of galaxies and is easier to apply to other observations and model predictions. Our main conclusions are summarized below:

1. We define clumps as discrete star-forming regions that individually contribute more than $8 \%$ of the rest-frame UV light of their galaxies. This definition is determined by comparing the fractional luminosity function of starforming regions, i.e., the number of star-forming regions per galaxy that contribute a given fraction of the UV luminosity of the galaxies, of real and redshifted nearby spiral galaxies. Clumps defined this way are significantly brighter than the redshifted $\mathrm{H}$ II regions of nearby large spiral galaxies and hence cannot be explained by the blending of the H II regions due to the decrease of physical spatial resolution and cosmological dimming.

2. Given the above definition, we measure the fraction of clumpy galaxies $\left(f_{\text {clumpy }}\right)$ in SFGs, requiring each clumpy galaxy to contain at least one off-center clump. The redshift 
evolution of the clumpy fraction changes with the $M_{*}$ of the galaxies. Low-mass galaxies $\left(\log \left(M_{*} / M_{\odot}\right)<9.8\right)$ keep a constant $f_{\text {clumpy }}$ of $\sim 60 \%$ from $z \sim 3$ to $z \sim 0.5$. Intermediate-mass galaxies $\left(9.8<\log \left(M_{*} / M_{\odot}\right)<10.6\right)$ keep their $f_{\text {clumpy }}$ almost a constant around $55 \%$ from $z \sim$ 3 to $z \sim 1.5$, and then gradually drops it to $40 \%$ at $z \sim 0.5$. Massive galaxies $\left(10.6<\log \left(M_{*} / M_{\odot}\right)<11.4\right)$ also keep their $f_{\text {clumpy }}$ constant at $\sim 55 \%$ from $z \sim 3$ to $z \sim 2$, but then quickly drop it to $\sim 15 \%$ at $z \sim 0.5$.

3. $f_{\text {clumpy }}$ decreases with $M_{*}$ at all redshift ranges, but the slope of the decrease changes with the redshift: the lower the redshift is, the faster the trend decreases. At $0.5<z<1.0$, $f_{\text {clumpy }}$ decreases from $60 \%$ at $\log \left(M_{*} / M_{\odot}\right) \sim 9.0$ to $15 \%$ at $\log \left(M_{*} / M_{\odot}\right) \sim 11.5$. At $1<z<2, f_{\text {clumpy }}$ decreases from around $60 \%$ at the lowest $M_{*}$ to $\sim 30 \%$ at $\log \left(M_{*} / M_{\odot}\right)>11.00$. At the highest redshift bin $z>2, f_{\text {clumpy }}$ only shows a mild decrease from $\sim 55 \%$ at $\log \left(M_{*} / M_{\odot}\right) \sim 9$ to $45 \%$ at $\log \left(M_{*} / M_{\odot}\right) \sim 10.5$.

4. $f_{\text {clumpy }}$ has important implications for the formation mechanisms of the clumps. We find that (1) the trend of disk stabilization predicted by VDI matches the $f_{\text {clumpy }}$ trend of massive galaxies; (2) minor mergers are a viable explanation of the $f_{\text {clumpy }}$ trend of intermediate galaxies at $z<1.5$, given a realistic observability timescale; and (3) major mergers are unlikely responsible for $f_{\text {clumpy }}$ in all masses at $z<1.5$. The roles of both minor and major mergers on low-mass galaxies at all redshifts or on intermediate-mass and massive galaxies at $z>1.5$ are still unclear due to the lack of a robust estimate of the merger rates at $z>1.5$.

5. We derive the clump contribution to the total UV luminosity of the galaxies $\left(C_{\mathrm{UV}}\right)$, taking into account the effects of clump-clump and clump-blob blending at high redshifts. At all redshifts, $C_{\mathrm{UV}}$ increases with the $M_{*}$ of the galaxies from $\log \left(M_{*} / M_{\odot}\right) \sim 9$ to $\log \left(M_{*} / M_{\odot}\right) \sim 10$ reaches a broad peak around $\log \left(M_{*} / M_{\odot}\right) \sim 10.5$, and then quickly drops. We speculate that the molecular gas fraction plays a major role on the trend of $C_{\mathrm{UV}}$ in intermediate and massive galaxies at least.

6. We convert $C_{\mathrm{UV}}$ into the clump contribution to the SFR of the SFGs $\left(C_{\mathrm{SFR}}\right)$, under an assumption that the dust extinction of clumps is lower than that of the galaxies. The redshift evolution of $C_{\mathrm{SFR}}$ shows mild trends at different $M_{*}$ ranges. For low- and intermediate-mass galaxies $(9<$ $\left.\log \left(M_{*} / M_{\odot}\right)<10.6\right), C_{\mathrm{SFR}}$ increases by almost a factor of two from $z \sim 3$ to $z \sim 0.5$. For massive galaxies $\left(\log \left(M_{*} / M_{\odot}\right)>10.8\right), C_{\mathrm{SFR}}$ shows a mild decrease from $z \sim 3$ to $z \sim 0.5$. We emphasize again that both our $C_{\mathrm{UV}}$ and $C_{\mathrm{SFR}}$ are the contributions to all SFGs rather than to only "clumpy" galaxies.

It is important to note that our clump definition is established from observations and does not incorporate any prior requirements from any theoretical models. For example, we do not ask our clumps to be gravitationally bound or formed in disks. The detachment of the observations and theories is important when we are still carrying out basic demographic studies of observational phenomena. It ensures that the demographic results would not be biased by any prevalent theoretical models, especially when the demographic results will be used to test the validity of those models.

It should also be noted that all our conclusions are based on off-center clumps. We do not include any clumps within $0.5 \times r_{e}$ of galaxies, which may bias our results. For example, the low $f_{\text {clumpy }}$ and low $C_{\mathrm{UV}}$ in high-redshift low-mass galaxies could be due to the neglect of clumps with small galactocentric distances, if clumps are preferentially formed or evolved into locations close to the galactic centers.

Our results are also only valid for $U V$-bright clumps. This is reasonable given the fact that clumps are traditionally and mostly identified in the rest-frame UV images (e.g., Elmegreen \& Elmegreen 2005; Elmegreen et al. 2007; Guo et al. 2012). Recent studies, however, also identify clumps from the restframe optical images (e.g., Elmegreen et al. 2009a; Förster Schreiber et al. 2011). These "red" clumps contain important clues to the fate of the clumps, namely, whether they would migrate toward the gravitational centers of their host galaxies, due to clump interactions and dynamical friction, and eventually coalesce into a young bulge as the progenitor of today's bulges (e.g., Elmegreen et al. 2008; Ceverino et al. 2010) or be quickly disrupted by either tidal force or stellar feedback to become part of a thick disk (e.g., Bournaud et al. 2009; Dekel et al. 2009b; Murray et al. 2010; Genel et al. 2012). To answer this question, a similar physical definition of clumps needs to be migrated to the red bands, and an accurate measurement of clump properties (e.g., $M_{*}$ age, SFR) is required.

We thank the anonymous referee for constructive comments that improve this article. Support for Program number HST-GO12060 and HST-GO-13309 were provided by NASA through a grant from the Space Telescope Science Institute, which is operated by the Association of Universities for Research in Astronomy, Incorporated, under NASA contract NAS5-26555. We acknowledge partial support from NSF grant AST 08-08133.

Facility: HST (ACS, WFC3)

\section{APPENDIX \\ COMPARISON BETWEEN OUR BLOB FINDER AND CANDELS VISUAL CLUMPINESS}

A sanity check of the efficiency and accuracy of our blob finder is to compare it with visual inspections. The CANDELS team has carried out a visual classification of galaxies with $H_{\mathrm{F} 160 \mathrm{~W}}<24.5 \mathrm{AB}$ mag in the both GOODS-S and UDS fields to determine the morphological class (i.e., spheroid, disk, irregular, etc.), interaction class (i.e., merger, interacting, nonmerger, etc.), clumpiness, etc. of the galaxies. Each galaxy has been inspected by 3-5 astronomers, mainly through its F160W images and complemented by its images in other bands. The details of the visual classification and the results are given by Kartaltepe et al. (2014). Here, we only use its visual clumpiness.

The scheme of visually determining clumpiness is shown in Figure 14. It starts from a $3 \times 3$ grid spanned by blue patchiness and major clumpiness. Each cell of the grid is given a score as the following:

0.0: no clumpy/no patches;

0.25: 1-2 clumps but no patches OR no clumps but some patches;

0.5: $3+$ clumps but no patches OR 1-2 clumps but some patches OR no clumps but lots of patches;

0.75: $3+$ clumps and some patches OR 1-2 clumps and lots of patches;

1.0: $3+$ clumps and lots of patches.

For each galaxy, each inspector can choose more than one option (cell) based on whether the galaxy has any blue patches (diffuse discrete regions based on the F606W image) and/or any major clumps (concentrated discrete regions 


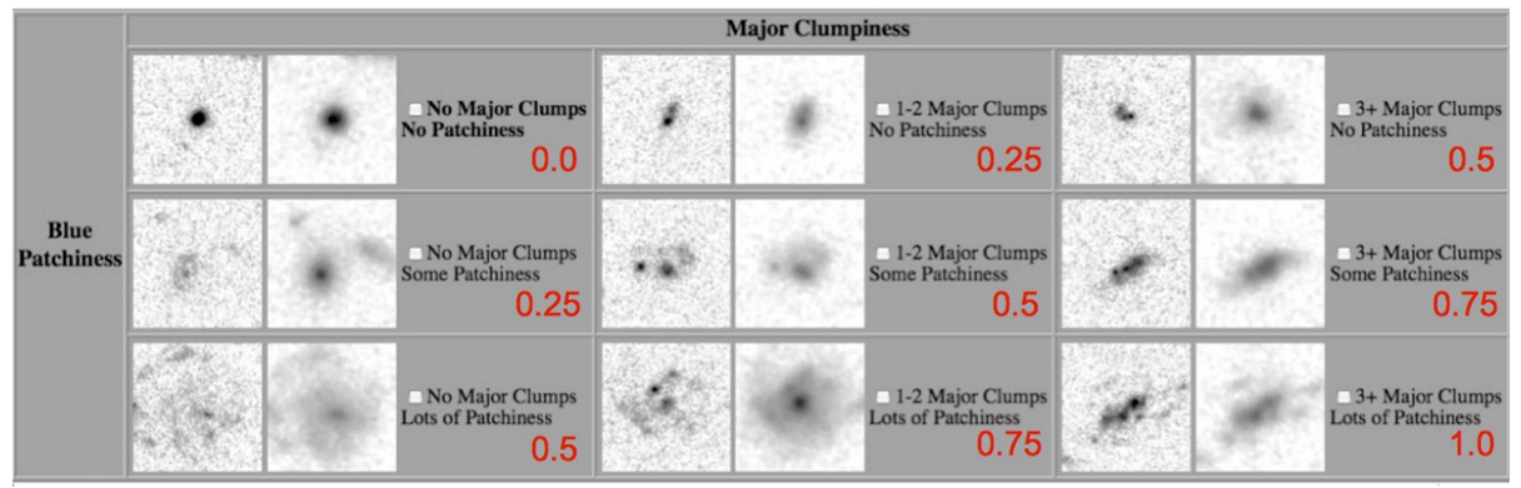

Figure 14. Scheme of the CANDELS visual classification of galaxy clumpiness. For each galaxy, each inspector can choose more than one option in the $3 \times 3$ matrix spanned by blue patchiness and major clumpiness. The score of each option (cell) is labeled by the red numbers. The scores of the selected options are then averaged to get the score of the inspector. The scores of all inspectors are then averaged to get a single score between 0 and 1 for each galaxy. For each option, the images are the F606W (left) and F160W (right) bands.
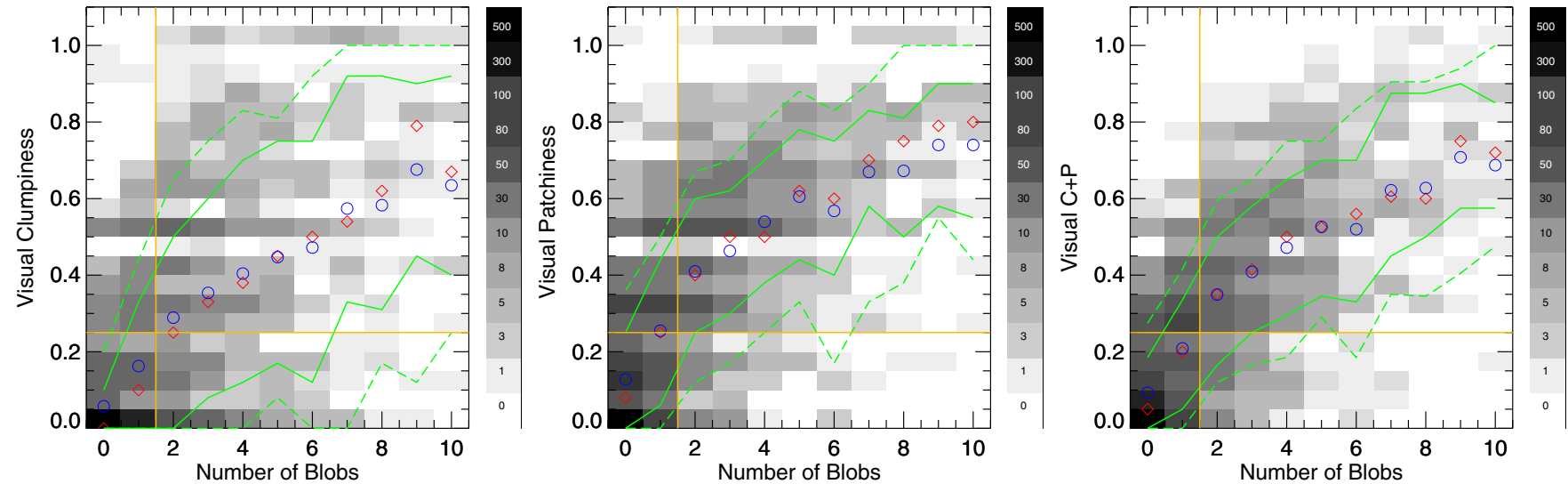

Figure 15. Comparisons of the CANDELS visual clumpiness (left), visual patchiness (middle), and visual clumpiness plus patchiness (right) with the number of blobs (bulge or galaxy center is excluded) detected by the automated blob finder. The gray scale shows the number of galaxies (as indicated in the gray-scale bar in the right side) in each bin. Red diamonds and blue circles show the mean and median visual clumpiness at given number of blobs. Green curves from bottom to top show the 10th, 20th, 80th, and 90th percentiles.

based on both F606W and F160W images). The scores of chosen cells are then averaged to a single score of the inspector. The single scores from all inspectors for the galaxy are averaged to get the final score between 0 and 1 of the galaxy. This value, visual clumpiness and patchiness $(\mathrm{VC}+\mathrm{P})$, includes both clumps and patches under the assumption that both are the same phenomenon and have the same physical nature (see Trump et al. 2014). This assumption is, however, under debate, as some astronomers believe that the clumps and the patches have different formation mechanisms. To provide a comprehensive comparison, we measure two other values from the visual classification. The first one is the score averaged over only the cells concerned in the clumpiness ( $\mathrm{Vi}$ sual Clumpiness or VC), and the second one is that averaged over only the cells concerned in the patches (Visual Patchiness or VP).

We compare the number of blobs (NB) of the GOODS-S galaxies in our sample with VC (left panel), VP (middle), and $\mathrm{VC}+\mathrm{P}$ (right) in Figure 15. There is good agreement (both median and mean) in all three panels, demonstrating that, in terms of detecting irregular star formation patterns, our blob finder works consistently with the CANDELS visual inspection. Interestingly, the scatter in $\mathrm{NB}$ versus $\mathrm{VC}+\mathrm{P}$ and $\mathrm{NB}$ versus $\mathrm{VP}$ is smaller than that in NB versus $\mathrm{VC}$, suggesting that our blob finder has better agreement with the visual values that contain patches. This is not too surprising, though, because our detection algorithm has no constraints on the concentration of blobs, while VC does. The implementation of such a concentration requirement is actually hard and uncertain in both automated and visual classifications, especially at high redshift, where the concentration measurement is difficult even for overall galaxies, let alone for the galactic sub-structures.

If we choose the visual values ( $\mathrm{VC}, \mathrm{VP}$, or $\mathrm{VC}+\mathrm{P}$ ) equal to 0.25 or the number of blobs (NB) equal to 1.5 (yellow lines in the figure) as the threshold of being a "clumpy" (more accurately, blobby) galaxy, the agreed classification rate is $\sim 75 \%$ for all $\mathrm{VC}, \mathrm{VP}$, and $\mathrm{VC}+\mathrm{P}$.

If we assume that our automated detection is correct, the fraction of the Type I error $(\mathrm{NB}<1.5$ and visual values $>0.25$, namely, the visual value falsely accepts a nonclumpy galaxy as a clumpy galaxy) and the Type II error (NB $>1.5$ and visual values $<0.25$, namely, the visual value falsely excludes a clumpy galaxy as a non-clumpy galaxy) show opposite behaviors for VC and VP. For VC, the Type I error $(10 \%)$ is less than the Type II error $(15 \%)$, while for VP, the Type I error (20\%) is larger than the Type II error (5\%). This interesting result gives us a guideline of using the VC and VP: if one wants a conservative sample only focusing on well defined clumps, VC should be used to reduce the Type I error, although it may miss some diffused clumps. On the other hand, if one wants a sample with as many as possible clumpy candidates, $\mathrm{VP}$ or $\mathrm{VC}+\mathrm{P}$ should be used to reduce the Type II error. 


\section{REFERENCES}

Barden, M., Jahnke, K., \& Häußler, B. 2008, ApJS, 175, 105 Barro, G., Faber, S. M., Perez-Gonzalez, P. G., et al. 2014, ApJ, 791, 52 Barro, G., Pérez-González, P. G., Gallego, J., et al. 2011, ApJS, 193, 30 Bigiel, F., Leroy, A., Walter, F., et al. 2008, AJ, 136, 2846 Bournaud, F., Daddi, E., Elmegreen, B. G., et al. 2008, A\&A, 486, 741 Bournaud, F., Daddi, E., Weiss, A., et al. 2014, arXiv:1409.8157 Bournaud, F., Dekel, A., Teyssier, R., et al. 2011, ApJL, 741, L33 Bournaud, F., Elmegreen, B. G., \& Martig, M. 2009, ApJL, 707, L1 Brammer, G. B., van Dokkum, P. G., Franx, M., et al. 2012, ApJS, 200, 13 Bruzual, G., \& Charlot, S. 2003, MNRAS, 344, 1000

Cacciato, M., Dekel, A., \& Genel, S. 2012, MNRAS, 421, 818

Calzetti, D., Armus, L., Bohlin, R. C., et al. 2000, ApJ, 533, 682

Calzetti, D., Kinney, A. L., \& Storchi-Bergmann, T. 1994, ApJ, 429, 582

Ceverino, D., Dekel, A., \& Bournaud, F. 2010, MNRAS, 404, 2151

Ceverino, D., Dekel, A., Mandelker, N., et al. 2012, MNRAS, 420, 3490

Chabrier, G. 2003, PASP, 115, 763

Conselice, C. J. 2003, ApJS, 147, 1

Conselice, C. J., Grogin, N. A., Jogee, S., et al. 2004, ApJL, 600, L139

Cowie, L. L., Hu, E. M., \& Songaila, A. 1995, AJ, 110, 1576

Cresci, G., Mannucci, F., Maiolino, R., et al. 2010, Natur, 467, 811

Daddi, E., Bournaud, F., Walter, F., et al. 2010, ApJ, 713, 686

Dahlen, T., Mobasher, B., Faber, S. M., et al. 2013, ApJ, 775, 93

Dekel, A., \& Birnboim, Y. 2006, MNRAS, 368, 2

Dekel, A., Birnboim, Y., Engel, G., et al. 2009a, Natur, 457, 451

Dekel, A., \& Burkert, A. 2014, MNRAS, 438, 1870

Dekel, A., Sari, R., \& Ceverino, D. 2009b, ApJ, 703, 785

Dekel, A., \& Silk, J. 1986, ApJ, 303, 39

Elmegreen, B. G., Bournaud, F., \& Elmegreen, D. M. 2008, ApJ, 688, 67

Elmegreen, B. G., \& Elmegreen, D. M. 2005, ApJ, 627, 632

Elmegreen, B. G., Elmegreen, D. M., Fernandez, M. X., \& Lemonias, J. J. 2009a, ApJ, 692, 12

Elmegreen, D. M., Elmegreen, B. G., Marcus, M. T., et al. 2009b, ApJ, 701, 306

Elmegreen, D. M., Elmegreen, B. G., Ravindranath, S., \& Coe, D. A. 2007, ApJ, 658,763

Elmegreen, D. M., Elmegreen, B. G., \& Sheets, C. M. 2004, ApJ, 603, 74

Erb, D. K., Steidel, C. C., Shapley, A. E., et al. 2006, ApJ, 646, 107

Förster Schreiber, N. M., Genzel, R., Bouché, N., et al. 2009, ApJ, 706, 1364

Förster Schreiber, N. M., Shapley, A. E., Genzel, R., et al. 2011, ApJ, 739, 45

Galametz, A., Grazian, A., Fontana, A., et al. 2013, ApJS, 206, 10

Gargiulo, A., Saracco, P., \& Longhetti, M. 2011, MNRAS, 412, 1804

Genel, S., Naab, T., Genzel, R., et al. 2012, ApJ, 745, 11

Genzel, R., Burkert, A., Bouché, N., et al. 2008, ApJ, 687, 59

Genzel, R., Newman, S., Jones, T., et al. 2011, ApJ, 733, 101

Giavalisco, M., Ferguson, H. C., Koekemoer, A. M., et al. 2004, ApJL, 600, L93

Giavalisco, M., Vanzella, E., Salimbeni, S., et al. 2011, ApJ, 743, 95

Grogin, N. A., Kocevski, D. D., Faber, S. M., et al. 2011, ApJS, 197, 35

Guo, Y., Ferguson, H. C., Giavalisco, M., et al. 2013, ApJS, 207, 24

Guo, Y., Giavalisco, M., Cassata, P., et al. 2011, ApJ, 735, 18

Guo, Y., Giavalisco, M., Ferguson, H. C., Cassata, P., \& Koekemoer, A. M. 2012, ApJ, 757, 120

Hopkins, P. F., Kereš, D., \& Murray, N. 2013, MNRAS, 432, 2639

Immeli, A., Samland, M., Gerhard, O., \& Westera, P. 2004a, A\&A, 413, 547

Immeli, A., Samland, M., Westera, P., \& Gerhard, O. 2004b, ApJ, 611, 20
Jones, T. A., Swinbank, A. M., Ellis, R. S., Richard, J., \& Stark, D. P. 2010 MNRAS, 404, 1247

Jonsson, P. 2006, MNRAS, 372, 2

Jonsson, P., Groves, B. A., \& Cox, T. J. 2010, MNRAS, 403, 17

Jonsson, P., \& Primack, J. R. 2010, NewA, 15, 509

Kartaltepe, J. S., Mozena, M., Kocevski, D., et al. 2014, arXiv:1401.2455

Kassin, S. A., Weiner, B. J., Faber, S. M., et al. 2012, ApJ, 758, 106

Kennicutt, R. C., Jr. 1998, ARA\&A, 36, 189

Kereš, D., Katz, N., Weinberg, D. H., \& Davé, R. 2005, MNRAS, 363, 2

Koekemoer, A. M., Faber, S. M., Ferguson, H. C., et al. 2011, ApJS, 197, 36

Kriek, M., van Dokkum, P. G., Labbé, I., et al. 2009, ApJ, 700, 221

Krumholz, M. R., \& Dekel, A. 2010, MNRAS, 406, 112

Laidler, V. G., Papovich, C., Grogin, N. A., et al. 2007, PASP, 119, 1325

Liu, G., Calzetti, D., Kennicutt, R. C., Jr., et al. 2013, ApJ, 772, 27

Liu, G., Koda, J., Calzetti, D., Fukuhara, M., \& Momose, R. 2011, ApJ, 735,63

Livermore, R. C., Jones, T., Richard, J., et al. 2012, MNRAS, 427, 688

López-Sanjuan, C., Le Fèvre, O., Tasca, L. A. M., et al. 2013, A\&A, 553, A78

Lotz, J. M., Jonsson, P., Cox, T. J., et al. 2011, ApJ, 742, 103

Lotz, J. M., Jonsson, P., Cox, T. J., \& Primack, J. R. 2010, MNRAS, 404, 590

Mandelker, N., Dekel, A., Ceverino, D., et al. 2014, MNRAS, 443, 3675

Moody, C. E., Guo, Y., Mandelker, N., et al. 2014, MNRAS, 444, 1389

Murata, K. L., Kajisawa, M., Taniguchi, Y., et al. 2014, ApJ, 786, 15

Murray, N., Quataert, E., \& Thompson, T. A. 2010, ApJ, 709, 191

Newman, A. B., Ellis, R. S., Bundy, K., \& Treu, T. 2012, ApJ, 746, 162

Noguchi, M. 1999, ApJ, 514, 77

Obreschkow, D., \& Rawlings, S. 2009, ApJL, 696, L129

Oke, J. B. 1974, ApJS, 27, 21

Overzier, R. A., Heckman, T. M., Tremonti, C., et al. 2009, ApJ, 706, 203

Peng, C. Y., Ho, L. C., Impey, C. D., \& Rix, H.-W. 2002, AJ, 124, 266

Puech, M. 2010, MNRAS, 406, 535

Rauch, M., Haehnelt, M., Bunker, A., et al. 2008, ApJ, 681, 856

Ravindranath, S., Giavalisco, M., Ferguson, H. C., et al. 2006, ApJ, 652, 963

Riess, A. G., Strolger, L.-G., Casertano, S., et al. 2007, ApJ, 659, 98

Robertson, B. E., \& Bullock, J. S. 2008, ApJL, 685, L27

Saintonge, A., Kauffmann, G., Wang, J., et al. 2011, MNRAS, 415, 61

Schechter, P. 1976, ApJ, 203, 297

Scoville, N. Z., Polletta, M., Ewald, S., et al. 2001, AJ, 122, 3017

Snyder, G. F., Lotz, J., Moody, C., et al. 2014, MNRAS, submitted (arXiv:1409.1583)

Steidel, C. C., Erb, D. K., Shapley, A. E., et al. 2010, ApJ, 717, 289

Swinbank, A. M., Smail, I., Longmore, S., et al. 2010, Natur, 464, 733

Szomoru, D., Franx, M., Bouwens, R. J., et al. 2011, ApJL, 735, L22

Tacconi, L. J., Genzel, R., Neri, R., et al. 2010, Natur, 463, 781

Tacconi, L. J., Genzel, R., Smail, I., et al. 2008, ApJ, 680, 246

Tacconi, L. J., Neri, R., Genzel, R., et al. 2013, ApJ, 768, 74

Tadaki, K.-i., Kodama, T., Tanaka, I., et al. 2014, ApJ, 780, 77

Toomre, A. 1964, ApJ, 139, 1217

Trump, J. R., Barro, G., Juneau, S., et al. 2014, ApJ, 793, 101

van den Bergh, S., Abraham, R. G., Ellis, R. S., et al. 1996, AJ, 112, 359

van der Wel, A., Bell, E. F., Häussler, B., et al. 2012, ApJS, 203, 24

van der Wel, A., Chang, Y.-Y., Bell, E. F., et al. 2014a, ApJL, 792, L6

van der Wel, A., Franx, M., van Dokkum, P. G., et al. 2014b, ApJ, 788, 28

Williams, J. P., de Geus, E. J., \& Blitz, L. 1994, ApJ, 428, 693

Wuyts, S., Forster Schreiber, N. M., Genzel, R., et al. 2012, ApJ, 753, 114

Wuyts, S., Förster Schreiber, N. M., Lutz, D., et al. 2011, ApJ, 738, 106

Wuyts, S., Förster Schreiber, N. M., Nelson, E. J., et al. 2013, ApJ, 779, 135 